\title{
IMMUNE NETWORK BEHAVIOR-II. FROM OSCILLATIONS TO CHAOS AND STATIONARY STATES
}

- Rob J. De Boer †

Theoretical Biology,

Utrecht University,

Padualaan 8, $3584 \mathrm{CH}$ Utrecht, The Netherlands

(E.mail.rdb@alive.biol.ruu.nl)

- Alan S. Perelson

$\dagger$ Theoretical Division,

Los Alamos National Laboratory,

Los Alamos, NM 87545, U.S.A.

(E.mail:asp@receptor.lanl.gov)

- IoAnnis G. KeVREKidis

Department of Chemical Engineering,

Princeton University,

Princeton, NJ 08544, U.S.A.

(E.mail:yannis@arnold.princeton.edu)

Two types of behavior have been previously reported in models of immune networks. The typical behavior of simple models, which involve B cells only, is stationary behavior involving several steady states. Finite amplitude perturbations may cause the model to switch between different equilibria. The typical behavior of more realistic models, which involve both $\mathrm{B}$ cells and antibody, consists of autonomous oscillations and/or chaos. While stationary behavior leads to easy interpretations in terms of idiotypic memory, oscillatory behavior seems to be in better agreement with experimental data obtained in unimmunized animals. Here we study a series of models of the idiotypic interaction between two B cell clones. The models differ with respect to the incorporation of antibodies, $\mathbf{B}$ cell maturation and compartmentalization. The most complicated model in the series has two realistic parameter regimes in which the behavior is respectively stationary and chaotic. The stability of the equilibrium states and the structure and interactions of the stable and unstable manifolds of the saddle-type equilibria turn out to be factors influencing the model's behavior. Whether or not the model is able to attain any form of sustained oscillatory behavior, i.e. limit cycles or chaos, seems to be determined by (global) bifurcations involving the stable and unstable manifolds of the equilibrium states. We attempt to determine whether such behavior should be expected to be attained from reasonable initial conditions by incorporating an immune response to an antigen in the model. A comparison of the behavior of the model with experimental data from the literature provides suggestions for the parameter regime in which the immune system is operating. 
1. Introduction. Jerne (1974) suggested that the lymphocytes of the immune system may be organized in the form of a network, with communication between cells occurring by idiotypic interactions. In order to understand the possible dynamic behavior of immune networks we have undertaken the study of one of the simplest networks, which consists of two clones activating each other by idiotypic interactions. Even though the network hypothesis is now close to 20 years old, this simple network has not been fully characterized. The models that we have proposed have steady states, which we have called immune states, in which the two B cell clones keep each other activated. These immune states may or may not be stable. In the first paper of this series (De Boer et al., 1993), which we hereafter call Part I, we have established that differences in the time scales of B cell and antibody turnover destabilize the immune states inducing limit cycle behavior. In this paper we study the effect of several other time scales involved in antibody secretion by $\mathrm{B}$ cells and loss of antibody from the site of antibody production.

The behavior of the model that we study is complicated. Our analysis, therefore, greatly benefits from the theoretical framework that we have built up in Part I by analysing two simplified models. This framework was derived by a bifurcation analysis of the steady states of the simplified (or basic) models. We have established the parameter conditions for existence and stability of the steady states. The invariant manifold structure of the saddle-type steady states proved to play a crucial role in the model behavior. Having this framework established, we now study more realistic versions of the model and compare the behavior of the model with experimental data. Natural antibody concentrations have been described to fluctuate periodically or chaotically (Lundkvist et al., 1989; Varela et al., 1991). We will compare the amplitude and the period of the fluctuations with those of our model. We will also study immune responses to antigens to see whether the attractors that we describe are attainable by external perturbation with antigens of the network in a quiescent state.

In most of the models studied thus far, one assumes that each B cell clone receives a constant input of virgin $\mathrm{B}$ cells from the bone marrow. It is uncertain whether the assumption of a constant source is correct. From the genetics of antibody $\mathrm{V}$ region recombination it seems that the potential repertoire is much greater than the repertoire of $\mathrm{B}$ cells expressed in an organism. Typical estimates of the potential repertoire are larger than $10^{10}$ specificities (Darnell et al., 1986; Berek and Milstein, 1988). Because a mouse has only $10^{8}$ B lymphocytes, probably comprising less than $10^{7}$ clones, it seems unlikely that the same $\mathrm{B}$ cell $\mathrm{V}$ region will be produced over and over again. Thus, we will test whether or not the model's behavior depends on a constant bone marrow source. 
2. The AB Model. In Part I we presented various forms of the antibody $B$ cell (AB) model. In our most complete $\mathrm{AB}$ model, the two-compartment AB model with gearing-up or CABG model, we consider B cells localized in the spleen. These cells may become activated by an anti-idiotypic antibody, upon which they proliferate and differentiate into antibody secreting cells. This maturation or gearing-up process takes a few days. Free antibody, produced by differentiated activated B cells, may activate anti-idiotypic B cells, may leave the spleen compartment and may react with anti-idiotypic antibodies in either the spleen or the circulation to form complexes. These complexes, which are analogous to antigen-antibody complexes, are known to be removed from the system by phagocytic cells such as macrophages. The rate of complex removal is one of the crucial parameters of the model.

The non-dimensionalized equations of the CABG model are given below. The derivation of these equations is explained in detail in Part I. We consider two $\mathrm{B}$ cell clones, $B_{1}$ and $B_{2}$, that activate each other by the antibodies, $A_{1}$ and $A_{2}$, which the $\mathrm{B}$ cells produce. The activation of $\mathrm{B}$ cells by an antibody is assumed to follow a log bell-shaped activation curve:

$$
f(A)=\frac{A}{\theta^{-1}+A} \frac{\theta}{\theta+A} .
$$

We consider two B cell populations inside the spleen:

$$
\begin{aligned}
& \frac{\mathrm{d} B_{1}}{\mathrm{~d} T}=\sigma+B_{1}\left(\rho f\left(A_{2}\right)-1\right), \\
& \frac{\mathrm{d} B_{2}}{\mathrm{~d} T}=\sigma+B_{2}\left(\rho f\left(A_{1}\right)-1\right) ;
\end{aligned}
$$

and two antibody concentrations inside the spleen:

$$
\begin{aligned}
& \frac{\mathrm{d} A_{1}}{\mathrm{~d} T}=\delta\left(B_{1} g_{1}-A_{1}\right)-\mu A_{1} A_{2}-\lambda\left(A_{1}-\hat{A}_{1}\right), \\
& \frac{\mathrm{d} A_{2}}{\mathrm{~d} T}=\delta\left(B_{2} g_{2}-A_{2}\right)-\mu A_{1} A_{2}-\lambda\left(A_{2}-\hat{A}_{2}\right) .
\end{aligned}
$$

Further, we consider two antibody concentrations in the blood outside the spleen:

$$
\frac{\mathrm{d} \hat{A}_{1}}{\mathrm{~d} T}=v \lambda\left(A_{1}-\hat{A}_{1}\right)-\delta \hat{A}_{1}-\mu \hat{A}_{1} \hat{A}_{2},
$$




$$
\frac{\mathrm{d} \hat{A}_{2}}{\mathrm{~d} T}=v \lambda\left(A_{2}-\hat{A}_{2}\right)-\delta \hat{A}_{2}-\mu \hat{A}_{1} \hat{A}_{2}
$$

and two "gearing up" equations accounting for the time lag involved in B cell maturation:

$$
\begin{gathered}
\frac{\mathrm{d} g_{1}}{\mathrm{~d} T}=\gamma\left(f\left(A_{2}\right)-g_{1}\right), \\
\frac{\mathrm{d} g_{2}}{\mathrm{~d} T}=\gamma\left(f\left(A_{1}\right)-g_{2}\right) .
\end{gathered}
$$

The representative parameter values that we use are $\theta=10, \delta=0.1, \sigma=$ $1.48 \times 10^{-3}, \rho=2, \mu=200, \lambda=5 \times 10^{4}, v=10^{-3}$ and $\gamma=0.4$. The justification of these parameter choices is described in detail in Part I.

In the companion paper we also proposed the symmetric logarithmic (SL) coordinate system for analysing basic versions of this model. Using a similar coordinate system for the CABG model is straightforward. First, we change to logarithmic variables $b_{i}^{*} \equiv \ln B_{i}, a_{i}^{*} \equiv \ln A_{i}, \hat{a}_{i}^{*} \equiv \ln \hat{A}_{i}$, for $i=1,2$. Second, we exploit the symmetry of the model by defining the differences and the sums of the $\log$ concentrations. Thus, we define $b_{\mathrm{d}} \equiv b_{1}^{*}-b_{2}^{*}, b_{\mathrm{s}} \equiv b_{1}^{*}+b_{2}^{*}, a_{\mathrm{d}} \equiv a_{1}^{*}-a_{2}^{*}$ and $a_{\mathrm{s}} \equiv a_{1}^{*}+a_{2}^{*}$, so that $b_{\mathrm{d}}=0$ whenever $b_{1}^{*}=b_{2}^{*}$, and $a_{\mathrm{d}}=0$ whenever $a_{1}^{*}=a_{2}^{*}$. The gearing up variables $g_{1}$ and $g_{2}$ vary between zero and one, and are not rescaled. Thus, we obtain for the activation function:

$$
f\left(e^{a}\right)=\frac{e^{a}}{\theta^{-1}+e^{a}} \frac{\theta}{\theta+e^{a}} ;
$$

for the B cells:

$$
\begin{gathered}
\frac{\mathrm{d} b_{\mathrm{d}}}{\mathrm{d} T}=\sigma\left(e^{-b_{1}^{*}}-e^{-b_{2}^{*}}\right)-\rho\left(f\left(e^{a_{1}^{*}}\right)-f\left(e^{a_{2}^{*}}\right)\right), \\
\frac{\mathrm{d} b_{\mathrm{s}}}{\mathrm{d} T}=\sigma\left(e^{-b_{1}^{*}}+e^{-b_{2}^{*}}\right)+\rho\left(f\left(e^{a_{1}^{*}}\right)+f\left(e^{a_{2}^{*}}\right)\right)-2 ;
\end{gathered}
$$

for the antibodies:

$$
\begin{gathered}
\frac{\mathrm{d} a_{\mathrm{d}}}{\mathrm{d} T}=\delta\left(g_{1} e^{b_{1}^{*}-a_{1}^{*}}-g_{2} e^{b_{2}^{*}-a_{2}^{*}}\right)-\mu\left(e^{a_{2}^{*}}-e^{a_{1}^{*}}\right)+\lambda\left(e^{\hat{a}_{1}^{*}-a_{1}^{*}}-e^{\hat{a}_{2}^{*}-a_{2}^{*}}\right), \\
\frac{\mathrm{d} a_{\mathrm{s}}}{\mathrm{d} T}=\delta\left(g_{1} e^{b_{1}^{*}-a_{1}^{*}}+g_{2} e^{b_{2}^{*}-a_{2}^{*}}-2\right)-\mu\left(e^{a_{2}^{*}}+e^{a_{1}^{*}}\right)+\lambda\left(e^{\hat{a}_{1}^{*}-a_{1}^{*}}+e^{\hat{a}_{2}^{*}-a_{2}^{*}}-2\right) ;
\end{gathered}
$$


for the peripheral antibody concentrations:

$$
\begin{aligned}
& \frac{\mathrm{d} \hat{a}_{1}^{*}}{\mathrm{~d} T}=v \lambda\left(e^{a_{1}^{*}-\hat{a}_{1}^{*}}-1\right)-\delta-\mu e^{\hat{a}_{2}^{*}}, \\
& \frac{\mathrm{d} \hat{a}_{2}^{*}}{\mathrm{~d} T}=v \lambda\left(e^{a_{2}^{*}-\hat{a}_{2}^{*}}-1\right)-\delta-\mu e^{\hat{a}_{1}^{*}} ;
\end{aligned}
$$

and for the gearing up variables:

$$
\begin{aligned}
& \frac{\mathrm{d} g_{1}}{\mathrm{~d} T}=\gamma\left(f\left(e^{a_{2}^{*}}\right)-g_{1}\right), \\
& \frac{\mathrm{d} g_{2}}{\mathrm{~d} T}=\gamma\left(f\left(e^{a_{1}^{*}}\right)-g_{2}\right) ;
\end{aligned}
$$

where $b_{1}^{*} \equiv\left(b_{\mathrm{d}}+b_{\mathrm{s}}\right) / 2, b_{2}^{*} \equiv\left(b_{\mathrm{s}}-b_{\mathrm{d}}\right) / 2, a_{1}^{*} \equiv\left(a_{\mathrm{d}}+a_{\mathrm{s}}\right) / 2$ and $a_{2}^{*} \equiv\left(a_{\mathrm{s}}-a_{\mathrm{d}}\right) / 2$.

3. Steady States. All equilibria that we have described in Part I for the basic model also exist in the CABG model. $\mathrm{B}$ cell proliferation is in equilibrium when $f(h) \approx 1 / \rho$, where the field, $h$, is either $A_{1}$ or $A_{2}$, see equations ( $2 \mathrm{a}$ ) and (b). Thus, using the approximation of $f(h)$ that we have introduced in Part I, one derives that the equilibrium antibody concentrations $A_{1}$ and $A_{2}$ are approximately $[\theta(\rho-1)]^{-1}$ and $\theta(\rho-1)$. For $\rho=2$ we obtain $A_{i} \approx \theta$ or $A_{i} \approx \theta^{-1}$, for $i=1,2$. This gives us the four activated states $\mathrm{MM}, \mathrm{HM}, \mathrm{MH}$ and $\mathrm{HH}$, where the state mnemonics are based on the level of concentration of the populations, i.e. $\mathrm{M}$ stands for moderate and $\mathrm{H}$ stands for high. (Either the $\mathrm{B}$ cell or antibody population level can be used, because at steady state high $\mathrm{B}$ cell concentrations imply high antibody concentrations and vice versa.) The virgin state $\mathrm{ZZ}$, for zero antibody concentrations, is the state $B_{1}=B_{2}=\sigma, A_{1}=A_{2}=0$ and hence $f\left(A_{1}\right)=f\left(A_{2}\right)=0$ and $A_{1}=A_{2}=G_{1}=G_{2}=0$.

Figure 1 illustrates the similarity between the basic $\mathrm{AB}$ model (called the $\mathrm{AB}$ model) and the CABG model with respect to both the steady states and the structure and interactions of some of the stable and unstable manifolds of the saddle-type steady states. The figure shows the original and the SL coordinate system. For the inverse spleen residence time $\lambda=10$ and the source rate $\sigma=0$ we show in Figs 1a and $\mathrm{c}$ the B cell nullclines. As was discussed in Part I these nullclines can be drawn because, for $\sigma=0$, the nontrivial B cell nullclines depend on antibody only. The nullclines in Figs $1 \mathrm{a}$ and $\mathrm{c}$ are very similar to those of the basic $\mathrm{AB}$ model presented in Figs $2 \mathrm{e}$ and $\mathrm{f}$ of Part I. In Fig. 1, i.e. for $\lambda=10$, the immune states, $\mathrm{HM}$ and $\mathrm{MH}$, are stable. The symmetric $\mathrm{MM}$ and $\mathrm{HH}$ states are usually unstable. The stable $\mathrm{ZZ}$ state does not appear in Fig. 1 
because the axes are logarithmic. The unstable manifolds of the symmetric MM and $\mathrm{HH}$ states are also very similar to those of the basic models. In Figs $1 \mathrm{~b}$ and $\mathrm{d}$ we show that the one-dimensional unstable manifold of the HH state is normal to the hyperplane of symmetry $A_{1}=A_{2}$ or $a_{\mathrm{d}}=0$, and asymptotically approaches the stable immune states (for $i=10$ ). The one-dimensional unstable manifold of the MM state lies on the hyperplane of symmetry, and one side asymptotically approaches the $\mathrm{HH}$ state, while the other side asymptotically approaches the virgin ZZ state.
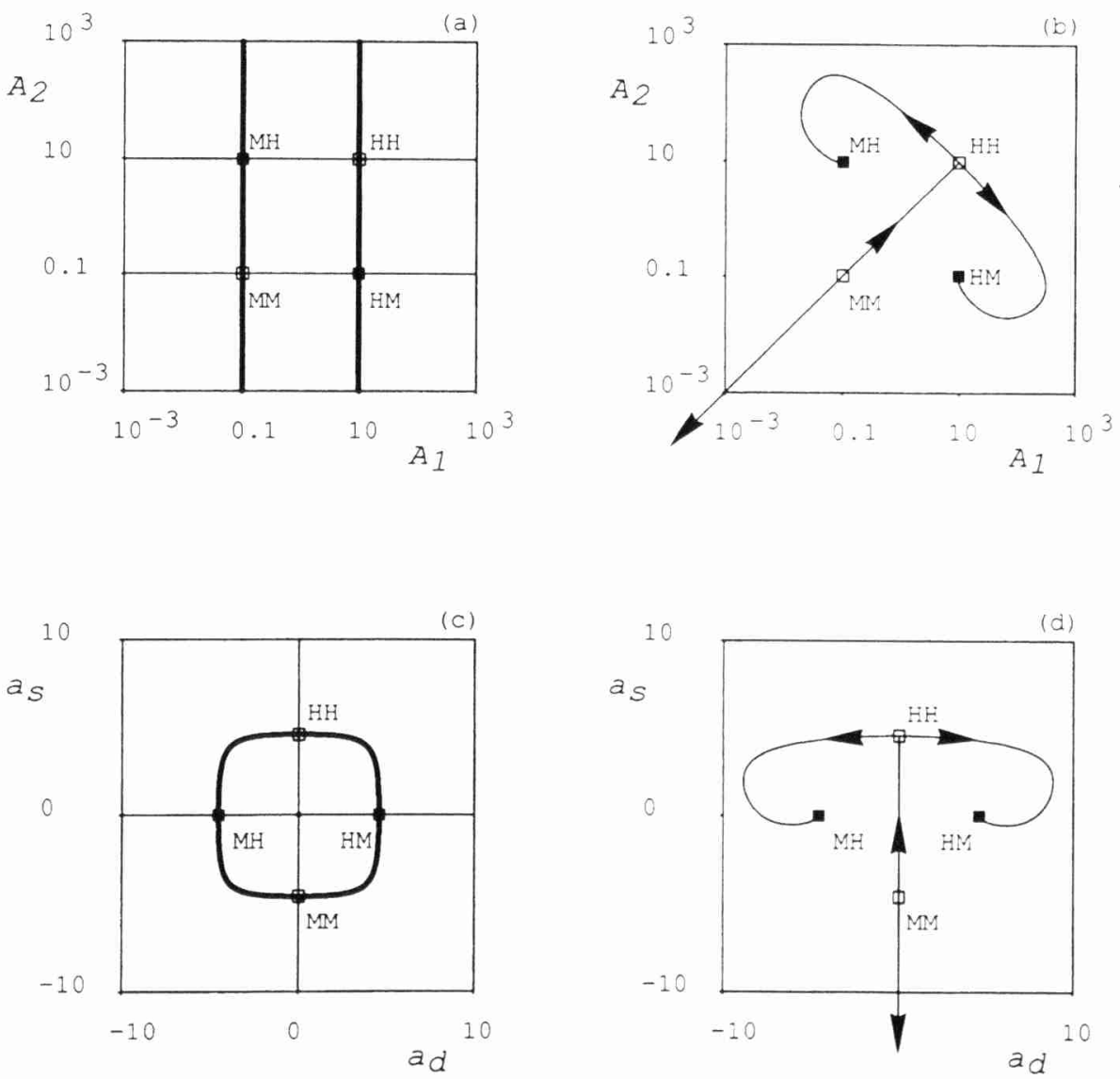

Figure 1. The steady states of the CABG model in the original and the SL coordinate system. The boxes (Z) denote equilibria: black means stable, open means unstable. Parameters: $i=10 . \theta=10 . \rho=2, \sigma=1.48 \times 10^{-3}, \delta=0.1, \mu=20$, $\eta=0.4$ and $v=10^{-3}$. (a) and (c) The nullclines. The heavy lines denote the $B_{2}^{\prime}=0$ and $b_{\mathrm{s}}^{\prime}$ nullclines. The light lines denote the $B_{1}^{\prime}=0$ and $b_{\mathrm{d}}^{\prime}$ nullclines. (b) and (d) The unstable manifolds of the MM and the $\mathrm{HH}$ state. The arrows indicate the direction of movement on the saddle insets and outsets. 
4. The Basic AB Model. We study the AB model in a series of steps. Thus, after we have analysed the basic $\mathrm{AB}$ model of Part I we increase the complexity of the model by first adding gearing up and then adding two compartments. The basic AB model is obtained from the CABG model by setting $\lambda=0$ and $\gamma \rightarrow \infty$, so that there is effectively only one compartment, the spleen, and no gearing up. For these parameter values $\hat{A}_{1}=\hat{A}_{2}=0$. Further, $G_{1} \rightarrow f\left(A_{2}\right)$ and $G_{2} \rightarrow f\left(A_{1}\right)$, see Part I.

4.1. Limit cycles and chaos. An overview of the dynamic behavior of the basic $\mathrm{AB}$ model is shown in Fig. 2, where we vary the complex formation parameter $0 \leqslant \mu \leqslant 15$ (for $\delta=0.1$ ). For these parameters the immune states are unstable when $\mu<12.6$. At $\mu \approx 12.6$ the immune states undergo a subcritical Hopf-bifurcation, see Fig. 6 of Part I. The dots in Figs $2 \mathrm{a}$ and $\mathrm{b}$ are values of $b_{\mathrm{d}}$ attained at a Poincaré section. After a long enough initial integration that should allow (fast) transients to "die out", we recorded 20 values of $b_{\mathrm{d}}$ at the intersection trajectories with the Poincare plane defined by $a_{\mathrm{d}}=0$. Thus, the Poincare section is a plane of symmetry. To include both periodic and chaotic solutions, this diagram was obtained using simulation forward in time and using zero-order continuation starting at a previous orbit. After 20 intersection points are recorded at one parameter value, a small change in the parameter $\mu$ is made and the system is allowed to approach a new attractor before the intersections with the Poincare plane are recorded again. We select the section hyperplane at the plane of symmetry $a_{\mathrm{d}}=0$, because oscillatory and chaotic trajectories typically cross this plane transversely repeatedly. We expect that the basic elements of the bifurcation structure are captured in the diagram, but because the numerics here are relatively coarse, some fine details may have
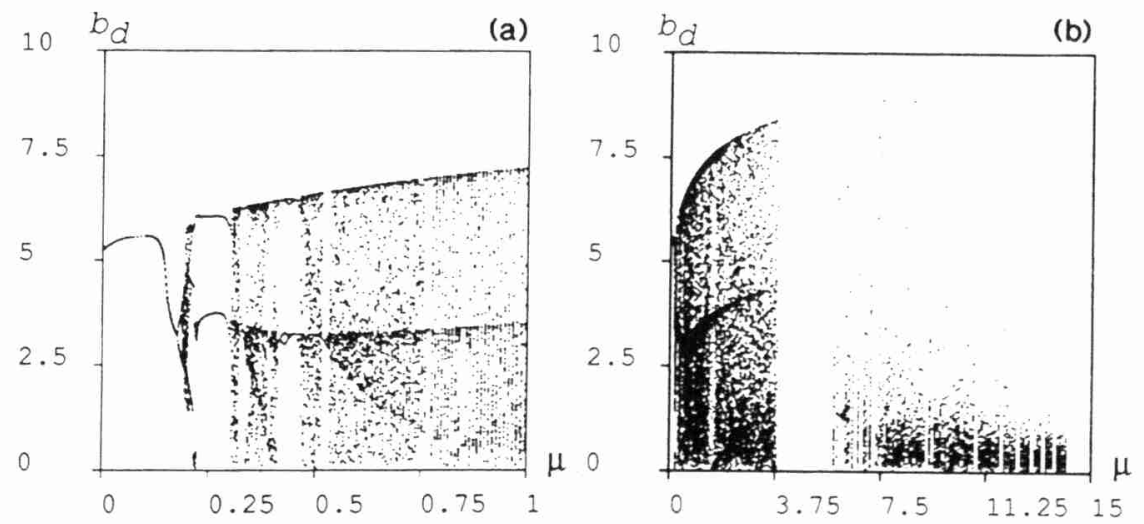

Figure 2. Bifurcation diagram of the basic AB model with respect to the parameter $\mu$. For each value of $\mu$ we plot $20 b_{\mathrm{d}}$ values attained at a Poincaré section at $a_{\mathrm{d}}=0$. Parameters: $\delta=0.1 . \theta=10, \rho=2$ and $\sigma=1.48 \times 10^{-3}$. (a) $0<\mu \leqslant 1$; (b) $0<\mu \leqslant 15$. 
been missed at this resolution. In selected cases below we have performed more careful continuations and stability calculations of limit cycles. In the chaotic regime we have checked for and located representative examples of type I intermittency.

Visual inspection of Fig. 2b seems to suggest four regions of behavior. In fact, there are only three regions, i.e. this visual observation is misleading. In the first region, i.e. $0 \leqslant \mu<3.7$, we have an apparently chaotic attractor that seems to be born via a period doubling cascade. In the second region, i.e. $3.7 \leqslant \mu<14$. we also have an apparently chaotic attractor (and complicated limit cycles in its periodic windows). The texture of this region is much lighter (and is even white for $3.75<\mu<6$, because our zero-order continuation has difficulties following the attractor). Below we will demonstrate, however, that the apparently chaotic attractor persists throughout this second region. The fact that the zeroorder continuation has these difficulties could be an indication of changes in the structure of the basin of attraction of the apparently chaotic attractor. This is studied in further detail below. In the third region, i.e. $\mu>14$, the apparently chaotic attractor has disappeared, and we find no further orbits intersecting the Poincaré plane.

In this third region the oscillatory chaotic behavior disappears around $\mu=14$ due to the subcritical Hopf bifurcation at $\mu \simeq 12.6$ after a small region of hysteresis. Apparently, the unstable limit cycles (and their stable manifolds) which are born at the subcritical Hopf bifurcation provide the basin boundary separating the stable HM and MH states and the apparent chaotic attractor in the interval of hysteresis, i.e. $12.6<\mu<14$. Note that in regions one and two, i.e. before the $\mathrm{HM}$ and $\mathrm{MH}$ states are stabilized through this subcritical Hopf bifurcation, the apparently chaotic attractor coexists with the stable ZZ state.

The basin of attraction of the sustained activated behavior of regions one and two is probably determined by the stable manifold of the MM state forming a separatrix between the trajectories that eventually approach the virgin state and those that do not. In the basic $\mathrm{AB}$ model the stable manifold of the MM state is a three-dimensional surface, since the MM state has three stable eigenvalues: one pair of complex eigenvalues and one real eigenvalue. Such a three-dimensional surface in four-dimensional space is too complex to visualize and analyse further. An alternative indication of changes in the structure and location of this three-dimensional surface is provided by the one-dimensional unstable manifold of the $\mathrm{HH}$ state. In the companion paper we presented global bifurcations involving the one-dimensional unstable manifold of the $\mathrm{HH}$ state and the stable manifold of the MM state. At the bifurcation points a heteroclinic connection was formed between the MM and $\mathrm{HH}$ state. Since the unstable manifold of the $\mathrm{HH}$ state is just one-dimensional it lends itself for further analysis and we use it as an indicator of the high-dimensional stable manifold of the MM state, and through that, of the basins of attraction. 
In Fig. 3 we show that for $\mu \approx 3$ we have a global bifurcation involving the unstable manifold of the $\mathrm{HH}$ state. This is shown in both the original and in the SL coordinate system. For $\mu \leqslant 3$, the one-dimensional unstable manifold of the $\mathrm{HH}$ state asymptotically approaches the apparently chaotic attractor for some complex oscillation within a periodic window lying close to it in phase space). In Figs $3 \mathrm{a}$ and $\mathrm{c}$ we illustrate for $\mu=3$ how this outset initially approaches the neighborhood of the MM state, and then asymptotically approaches a chaotic attractor winding around the immune states. For $\mu \geqslant 3.1$ (Figs $3 \mathrm{~b}$ and $\mathrm{d}$ ) the outset of the HH state spirals around the MM state and ultimately approaches the virgin state. For $3<\mu<3.1$ we again expect to find a global bifurcation point where the $\mathrm{HH}$ and the MM states are involved in a heteroclinic connection.

Similar to using the one-dimensional unstable manifold of the $\mathrm{HH}$ state as an indicator of the basin of attraction. we have also studied the two-dimensional unstable manifolds of the $\mathrm{HM}$ and $\mathrm{MH}$ states. In this region, i.e. for $\mu<12.6$, the largest eigenvalues of these asymmetric states consist of a complex pair with a positive real part. The two other eigenvalues are real and negative. The unstable manifold corresponding to these states is therefore two-dimensional. Using SCIGMA (Taylor et al., 1990) such planar manifolds can be studied by judiciously selecting a number of initial conditions on an ellipse surrounding the steady state on the local unstable eigenspace. We found that for $\mu \leqslant 3.6$ all of these trajectories asymptotically approach the chaotic attractor. Thus, in this region the unstable manifold of the asymmetric state asymptotically approaches the chaotic attractor. Conversely, for $\mu \geqslant 3.7$ the unstable manifold of HM and MH does not asymptotically approach a single attractor: typically, some trajectories on it will approach the virgin $\mathrm{ZZ}$ state, while other very nearby trajectories on it will asymptotically approach the chaotic attractor, or stable limit cycles in its periodic windows (e.g. for $\mu=6$ ). Thus, around $\mu=3.7$ we have a global bifurcation involving the unstable manifold of the asymmetric $\mathrm{HM}$ and $\mathrm{MH}$ states.

We believe that these two global bifurcations, i.e. the heteroclinic connection between the $\mathrm{HH}$ and the MM state at $\mu \approx 3$ and the bifurcation involving the unstable manifold of the $\mathrm{HM}$ and $\mathrm{MH}$ states at $\mu \approx 3.6$, significantly affect the size and structure of the basins of attraction in the model. since zero-order continuation may often "miss" the chaotic attractor. This accounts for the "lighter texture" of the chaotic regime for $3.7<\mu<14$ in Fig. 2b. Note that both global bifurcations probably involve the stable manifold of the MM state.

In Figs $3 e$ and $\mathrm{f}$, for $\mu=3.7$ (i.e. around the bifurcation point), we show two trajectories on the two-dimensional unstable manifold of the HM state. One trajectory seems to approach the apparently chaotic attractor, whereas the other can be seen to approach the $\mathrm{ZZ}$ state. In Fig. $3 \mathrm{f}$ we show a blow up of Fig. 3e around the MM state. Because both trajectories appear to approach the 
(a)

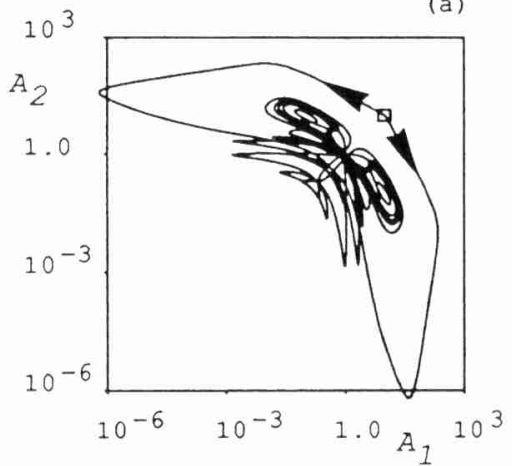

(c)

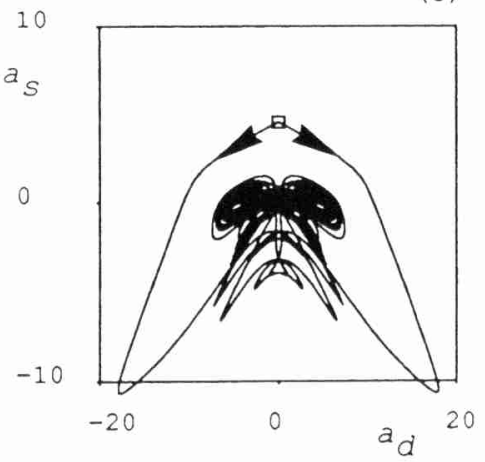

(e)

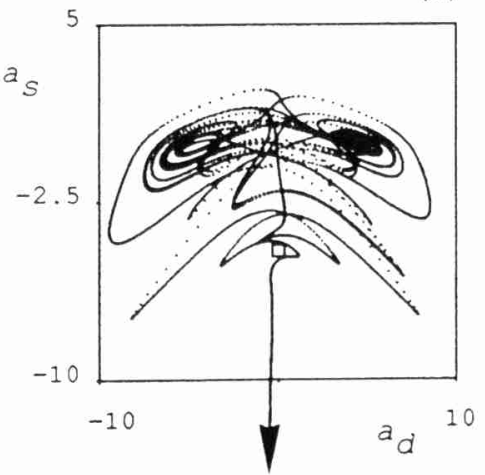

(b)

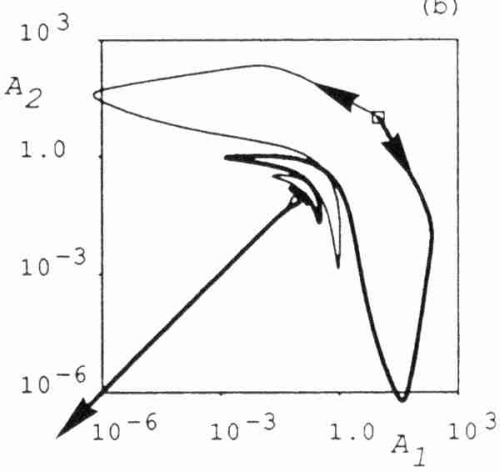

(d)

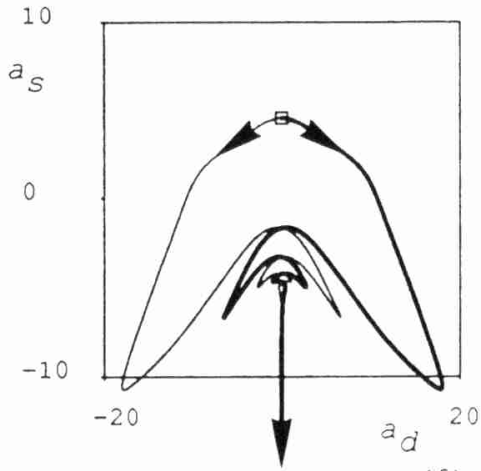

(f)

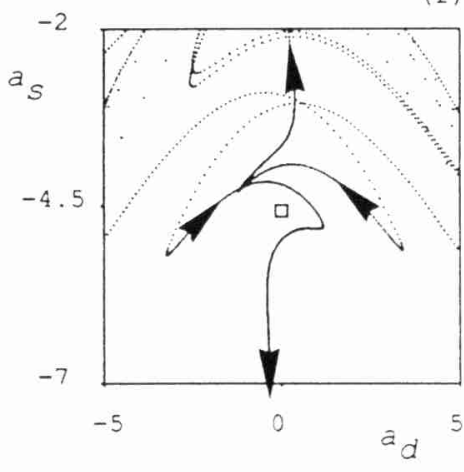

Figure 3. The unstable manifolds of the $\mathrm{HH}$ and the $\mathrm{HM}$ states of the basic $\mathrm{AB}$ model. The manifolds first approach the MM state spiraling and then turn either towards higher or lower antibody values. Parameters: $\delta=0.1, \theta=10, \rho=2$ and $\sigma=1.48 \times 10^{-3}$. In panels (a)-(d) the HH state is indicated by a square. Panels (a) and (b) and (c) and (d) differ only in the coordinate system. (a) and (c) $\mu=3$ : the $\mathrm{HH}$ outset asymptotically approaches the chaotic attractor. (b) and (d) $\mu=3.1$ : the $\mathrm{HH}$ outset asymptotically approaches the virgin state. In panels (e) and (f) the MM state is indicated by a square. (e) $\mu=3.7$ : one trajectory on the 20 unstable manifold of the HM state approaches the chaotic attractor, another asymptotically approaches the virgin state. (f) A blow up of panel (e) around the MM state. 
neighborhood of the MM state, it is conceivable that this bifurcation also involves the stable manifold of the MM state. The diversity of the ultimate fate of very nearby trajectories on the unstable manifolds of $\mathrm{HM}$ and $\mathrm{MH}$ strongly suggests a fractal basin boundary between the chaotic attractor and the $\mathrm{ZZ}$ state, and further suggests that the HM and MH steady states lie on this complex boundary. We are currently trying to elucidate the geometry of this boundary.

Examining the two regions where we find sustained behavior, we observe in Fig. 2 that starting at $\mu=0$ the model has a symmetric limit cycle that first increases, and then decreases in amplitude. We call a limit cycle symmetric when the two halves at each side of the lines of symmetry $a_{\mathrm{d}}=0$ and $b_{\mathrm{d}}=0$ are mirror images. For each population this means that its behavior during one half of the period is the same as the behavior of the other population during the other half of the period. This is sometimes referred to as "ponies on a merry-goround" (Aronson et al., 1991). At $\mu \approx 0.178$ the symmetric cycle goes through a pitchfork bifurcation, giving rise to two asymmetric cycles that are mirror images of each other. The asymmetric cycles undergo a period doubling cascade starting at $\mu \approx 0.18$. The fact that we find period doublings only after the symmetry breaking is in agreement with the arguments in Swift and Wiesenfeld (1984). This period doubling cascade apparently leads to chaos. with the classical periodic windows for some parameter values in the chaotic regime (see e.g. $0.23<\mu<0.3$, and Fig. 4 d)

In Figs $4 \mathrm{a}-\mathrm{e}$ we provide some samples of the model behavior taken from the overview in Fig. 2 before the global bifurcation involving the unstable manifold of the $\mathrm{HH}$ state at $\mu=3.7$. Similar examples in the original coordinate system can be found in De Boer and Perelson (1991). In Fig. 4a we show the two asymmetric limit cycles born in the pitchfork bifurcation at $\mu=0.178$ in a B cell. i.e. $b_{\mathrm{d}}$ and $b_{\mathrm{s}}$ phase space. After the period doubling cascade, i.e. for $\mu=0.2$, we find an apparently chaotic attractor as shown in Fig. 4b. In the next panels of Fig. 4 we show the model behavior in the antibody phase space and we show the $\mathrm{B}$ cell nullclines. We observed intermittency at $\mu=0.2235$ (Fig. 4c). The intermittency is of type I (Pomeau and Manneville, 1980) and is analysed in further detail in Fig. 5. In Fig. 4d we show one of two asymmetric limit cycles that exists in a periodic window at $\mu=0.25$. In Fig. 4 e we illustrate for $\mu=2$ how the chaotic attractor resembles the Lorenz "butterfly" (Lorenz, 1963; Sparrow, 1982). This resemblance is due to the similarity in the framework of the two models. Both have a plane of symmetry separating two equilibria that switch from being a sink to being a source at a Hopf bifurcation.

In the second region, i.e. for $3.7<\mu<14$, we also find chaos with periodic windows. Figure $4 \mathrm{f}$ focuses on the region $\mu \approx 12.6$, where the immune state changes stability by the subcritical Hopf bifurcation (at which an unstable limit cycle is born towards higher values of $\mu$ ). In this region we observe hysteresis 

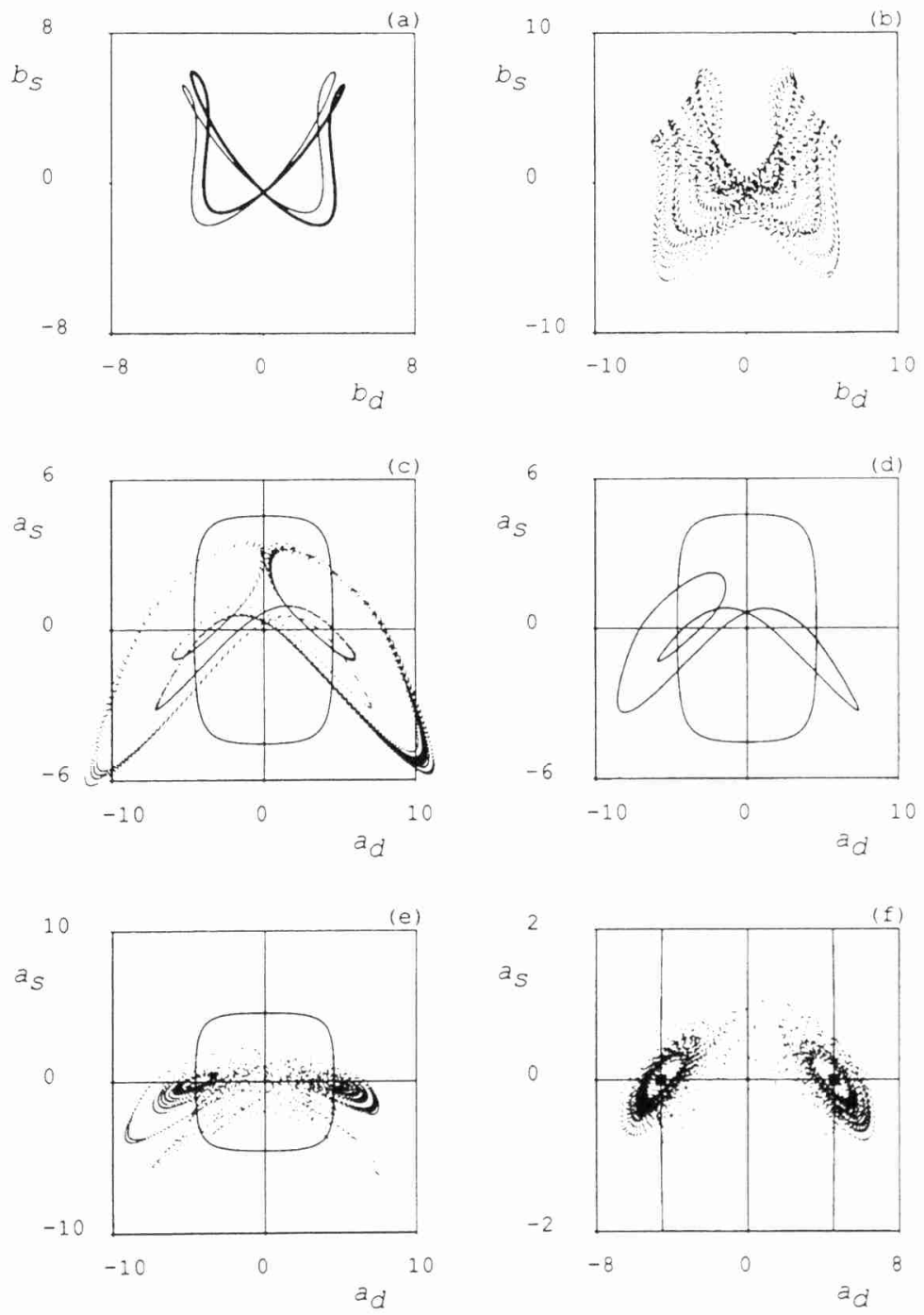

Figure 4. Illustrative phase portraits of the basic AB model. Parameters: $\delta=0.1$, $\theta=10, \rho=2$ and $\sigma=1.48 \times 10^{-3}$. Panels ( $\mathrm{a}$ ) and $(\mathrm{b})$ display the model behavior in a $b_{\mathrm{d}}$ vs $b_{\mathrm{s}}$ phase space. Panels (c)-(f) display the model behavior in an $a_{\mathrm{d}}$ vs $a_{\mathrm{s}}$ phase space. The light lines in panels (c) (f) are the $b_{\mathrm{d}}^{\prime}=0$ and $b_{\mathrm{s}}^{\prime}=0$ nullclines (for $\sigma=0$ ). (a) $\mu=0.18$ : the light and the heavy trajectory depict two asymmetric stable limit cycles. With respect to the plane of symmetry the limit cycles are mirror images. (b) $\mu=0.2$ : chaotic behavior. (c) $\mu=0.2235$ : intermittency, the trajectory makes many turns around one of two (unstable) limit cycles until it crosses to the other. (d) $\mu=0.25$ : a periodic window in which we show one of two asymmetric limit cycles. (e) $\mu=2$ : chaotic behavior around the immune states. This resembles qualitatively the "mask" underlying the Lorenz attractor. (f) $\mu=13$ : hysteresis, the chaotic attractor co-exists with two stable immune states (and a stable virgin state). 

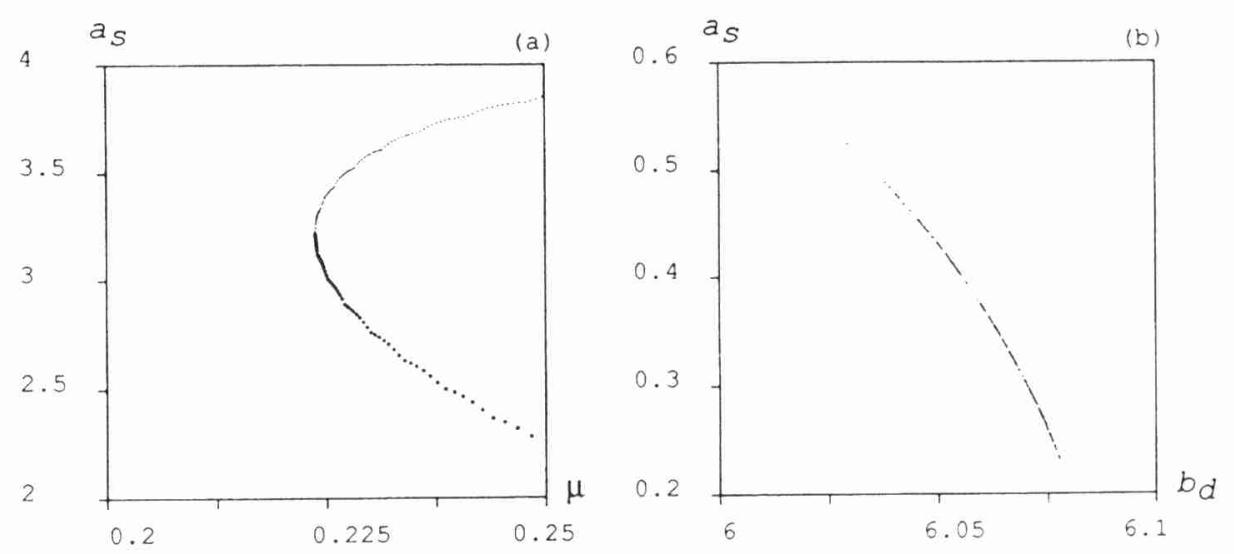

Figure 5. Intermittency in the basic AB model. Parameters: $0 \leqslant \mu \leqslant 0.25 . \delta=0.1$. $\theta=10 . \rho=2$ and $\sigma=1.48 \times 10^{-3}$. (a) A turning point at $\mu \simeq 0.22376$ in a continuation of the limit cycle shown in Fig. $4 \mathrm{~d}$. The heavy dots denote stable limit cycles, the light dots unstable ones. (b) A Poincaré map for $\mu=0.223$. showing values of $b_{\mathrm{d}}$ and $a_{\mathrm{s}}$ attained at the hyperplane $a_{\mathrm{d}}=0$. The slight curvature is characteristic for the "slowing down" of intermittency.

because the apparently chaotic attractor co-exists with two stable immune states (and, as always, one stable virgin state). The unstable limit cycles around the immune states. and their stable manifolds, define the basins of attraction of the immune states. Increasing $\mu$ increases the amplitude of the unstable limit cycles (De Boer et al., 1990). Around $\mu=14.2$ the chaotic attractor disappears. We suspect that its death is due to a global interaction with the chaotic attractor involving the growing unstable saddle-type limit cycles and their stable and unstable manifolds. This is supported by the observation of long "chaotic" transients, with unpredictable winding patterns around the neighborhood of the two limit cycles, which, for $\mu \geqslant 14.2$, eventually settle into one of the immune states or approach the virgin state.

4.2. An example of intermittency. Figure 5a represents the continuation of the asymmetric limit cycle shown in Fig. 4d towards the regime where intermittency was computationally observed. Indeed, a turning point bifurcation of limit cycles is observed for $\mu \simeq 0.22376$, above which the behavior is periodic, but below which it is type I intermittent (Pomeau and Manneville, 1980). This is illustrated in Fig. 5 b by the typical curvature of the Poincare map taken close to the turning point. Thus, for $\mu=0.223$, i.e. at a point where the limit cycles have "just" disappeared, we plot values of $b_{\mathrm{d}}$ and $a_{\mathrm{s}}$ attained at a large number of intersections of one trajectory with the Poincare plane defined by $a_{\mathrm{d}}=0$. Iterates on the Poincare map "slow down" when they approach this 
region of phase space. These repeated similar oscillations represent the quiescent regions between "bursts".

4.3. Oscillations in the absence of a source. A new and important feature of attractor in the region $6<\mu<14.1$ is that the model's behavior has become qualitatively independent of the source, $\sigma$, of novel B cells from the bone marrow. Thus, if we set $\sigma=0$ we obtain very similar behavior, i.e. oscillations, chaos and a subcritical Hopf bifurcation with hysteresis. From an immunological point of view this is significant because it is not clear that the bone marrow will regularly produce the same idiotype again (see also the Discussion in Part I). It is not clear, however, whether or not this behavior with $\sigma=0$ will be observable in experiments because the basin of attraction of the attractor often appears to be relatively small. Of course, in so many dimensions it is impossible to visualize the basins of attraction, and therefore they cannot be shown. Using zero-order continuation (making small steps in $\mu$ ) it is difficult to remain on the attractor. This can be taken to mean that many physically realistic initial conditions might not lie in its basin of attraction. This will be further discussed below.

We conclude that in this parameter region the basic $\mathrm{AB}$ model (i.e. with $\lambda=0$ and $\gamma \rightarrow \infty$ ) displays chaotic behavior with all of its classic phenomena (i.e. period doublings, periodic windows, intermittency). The model also exhibits a small region of hysteresis where chaotic behavior co-exists with stable immune steady states. Additionally, for $\mu>6$, the oscillatory/chaotic behavior of the model persists even if the bone marrow production is set to zero, i.e. $\sigma=0$. Lastly, the stationary states of the basic AB model are stable for $\mu>12.6$ (see Part I).

4.4. Antigens and memory: Mapping the various basins of attraction in high dimensional models is practically impossible. Nevertheless, we have some indications of bifurcations involving the stable manifold of the MM state which may influence the attainability of the attractors. To study this further we tried to see where "realistic" initial conditions would be attracted to. If certain coexisting attractors frequently attract the trajectories starting from more realistic initial conditions, we may conclude that the corresponding behaviors are more likely to be exhibited by the immune system.

In order to be able to define realistic initial conditions we will study an immune response to a simple, non-growing antigen. Thus, consider a molecular antigen, $P$ (e.g. a protein), recognized by $B_{1}$ cells and $A_{1}$ antibodies. We propose the same scaling for the antigen concentration as for the antibodies (see Part I), i.e. $P=1$ is the maximally stimulatory concentration. Assume that the antigen is removed from the system by the formation of antigen-antibody 
complexes. Incorporation of the antigen leads to new equations for the activation of $B_{1}$ cells because the field $h$ now consists of $A_{2}$ plus $P$ :

$$
\begin{gathered}
f(h)=\frac{h}{\theta^{-1}+h} \frac{\theta}{\theta+h}, \\
\frac{\mathrm{d} B_{1}}{\mathrm{~d} T}=\sigma+B_{1}(\rho f(h)-1) .
\end{gathered}
$$

where $h=A_{2}+P$. Furthermore, we need an additional term in the antibody equation for the $A_{1}$ antibodies because they may form antigen-antibody complexes:

$$
\frac{\mathrm{d} A_{1}}{\mathrm{~d} T}=\delta\left(B_{1} f(h)-A_{1}\right)-\mu A_{1} A_{2}-\kappa A_{1} P
$$

where $\kappa$ is the non-dimensional rate at which antigen-antibody complexes are removed. For the antigen we propose the simple equation:

$$
\frac{\mathrm{d} P}{\mathrm{~d} T}=-\delta_{P} P-\kappa A_{1} P \text {. }
$$

where $\delta_{P}$ is the normal turnover of the antigen. We make the turnover of antigen very small, i.e. $\delta_{P}=0.01$, in order to have prolonged activation of the system and to let antigen removal be specific. The antigen is introduced at a dose at which it is maximally stimulatory. i.e. $P(0)=1$, and the system is assumed to be in the virgin state, i.e. $B_{1}=B_{2}=\sigma$ and $A_{1}=A_{2}=0$. This activates the system and leads to the elimination of the antigen by the $A_{1}$ antibody. [Similar behavior is obtained when the antigen dose slightly deviates from $P(0)=1$.] The question that we ask is: when the network in its virgin state is stimulated with a maximally stimulatory dose of antigen, does the system remain activated or does it return to the virgin state? This can easily be determined by the antibody concentrations, which approach zero if the system returns to the virgin state but remain of the order one when the system remains activated. Note that the long-term solution of equation (14) is zero and, therefore, all the steady states, and their stability, and all the long-term behavior of the system is exactly the same as before.

We have studied this model for $k=10^{-2}, 10^{-3}$ and $10^{-4}$. For these values of $\kappa$ the system returns to the virgin state following the elimination of the antigen whenever $\mu>3.08$. For $\mu \leqslant 3.08$ the system attains sustained oscillatory/chaotic behavior (not shown). Since the critical value of $\mu \approx 3$ is located around the heteroclinic connection between the $\mathrm{HH}$ and the MM states, we conclude that the fate of the unstable manifold of the $\mathrm{HH}$ state provides an indication of the basin of attraction of the sustained behavior. 
Further, we might argue that because the critical value of $\mu \approx 3.08$ corresponds more closely to the bifurcation involving the unstable manifold of the $\mathrm{HH}$ state (around $\mu \approx 3$ ) than to that involving the unstable manifolds of the $\mathrm{HM}$ and $\mathrm{MH}$ states (around $\mu \approx 3.7$ ), the former bifurcation is more informative in predicting whether or not the sustained behavior is attained following stimulation with an antigen. Thus, we conjecture that the oscillatory/chaotic behavior found in the window $3.7<\mu<14$ is unlikely to be attained by an antigen-driven immune response. The same seems to be true for the stable immune states found for $\mu>12.6$. They apparently have so "small" a basin of attraction that they are unlikely to account for the memory of an encounter with an antigen. This will be discussed further below when we study the CABG model.

5. The AB Model with Gearing Up (ABG Model). Activated B cells produce antibodies and proliferate. The two processes correspond to two different stages of B cell differentiation. Typically a resting B cell when activated first enlarges and then goes through several rounds of cell division before it produces significant quantities of antibodies (Andersson et al. 1977). Even though $\mathrm{B}$ cells producing natural IgM antibodies are not involved in extensive clonal amplification (Coutinho et al., 1990), we still expect a delay between stimulation and antibody secretion. We model the fact that antibody production follows B cell activation with a time lag by using the gearing up function proposed by Segel and Perelson (1989).

We first incorporate gearing up into the basic $\mathrm{AB}$ model for which $i=0$, $\hat{A}_{1}=\hat{A}_{2}=0$. The resulting model consists of six ODEs and we call it the $A B$ model with gearing up or the ABG model. As is easy to see from equations (3) and (5), including gearing up does not affect the number of steady states found in the basic AB model.

We examine the effect of gearing up on the stability of the immune states in Fig. 6. The heavy line in Fig. 6a depicts the two-parameter continuation in $\mu$ and $\gamma$ of the Hopf bifurcation that we found for the immune state at $\mu=12.6$ and $\delta=0.1$. The immune state is stable above the heavy line and unstable below it (as is indicated by the shading). As $\mu$ decreases towards $\mu=12.6$, the twoparameter curve turns toward $; \rightarrow x$. This shows how the ABG model reduces to the basic $\mathrm{AB}$ model. The minimum value of $\gamma$ attained in the continuation is $\gamma=4.2$. The value $\gamma=0.4$, which is our empirical estimate for the rate of the gearing up process, is indicated by a horizontal line. For this value of $;$ the immune states are unstable. In Fig. 6 b we show several representative twoparameter continuations of the Hopf bifurcation for various values of the antibody lifetime, i.e. for $\delta=0.01,0.1,0.2,0.6$ and 1 , respectively. As indicated in Fig. 6b, for our estimate $\gamma=0.4$ we expect the immune state to be unstable for almost all values of $\mu$. The immune state is only stable when $\delta$ is (unrealistically) 

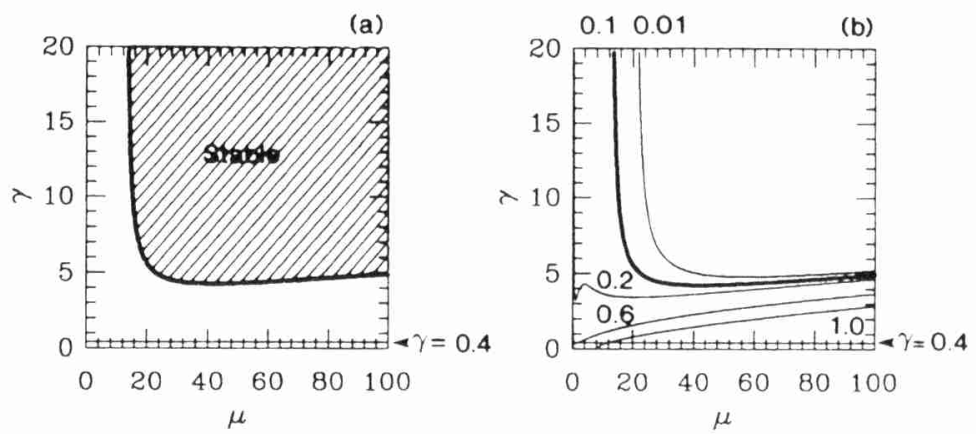

Figure 6. The gearing up $\mathrm{AB}$ model. A two-parameter diagram of the Hopf bifurcation, varying $\mu$ and $; ;$ as bifurcation parameters. Parameters: $\theta=10, \rho=2$, $\sigma=1.48 \times 10^{-3}, \delta=0.1$ and $i=y^{\prime}=0$. The horizontal line. $\gamma=0.4$. represents a reasonable value of the gearing up delay. That line falls well within the unstable regime. (a) A continuation for $\delta=0.1$. We have shaded the area in which the immune states are stable. (b) The heavy line is $\delta=0.1$. The light lines are continuations for $\delta=0.01,0.2,0.6$ and $\delta=1$.

large and $\mu$ is (unrealistically) small. We conclude that gearing up has a destabilizing effect on the immune steady states, especially in the realistically large $\mu$ parameter regime.

In Fig. 7 we provide an overview of the dynamic behavior of the gearing up $\mathrm{AB}$ model as a function of the rate of complex elimination $\mu$ using Poincare sections similar to those presented in Fig. 2. For each value of $\mu$ we plot the value of $b_{\mathrm{d}}$ attained at a Poincare section defined by the plane of symmetry, $a_{\mathrm{d}}=0$. We study the model in the unstable regime by setting ${ }_{i}=0.4$ and $\delta=0.1$. In this parameter region the only stable state is the virgin $\mathrm{ZZ}$ state.

For low values of $\mu$ the behavior of the $\mathrm{ABG}$ model is very similar to that of the basic AB model, see Fig. 7a. Starting at $\mu=0$ we see a symmetric limit cycle growing and decreasing in amplitude, going through a symmetry breaking bifurcation at $\mu \approx 0.51$. The two asymmetric cycles are mirror images with respect to the planes of symmetry $a_{\mathrm{d}}=0$ and $b_{\mathrm{d}}=0$. The asymmetric cycles go through a cascade of period doublings around $\mu=0.55$. The Feigenbaum cascade develops into chaotic behavior with periodic windows. Surprisingly, we find chaotic and periodic windows co-existing with the symmetric limit cycle even before the symmetry breaking, i.e. before $\mu \approx 0.51$. This is another region with hysteresis. Since the basin of attraction of the chaotic attractor in this region seems to be very small, this need not be very significant.

Similar to what was shown in Fig. 2, and in Fig. 6 of Part I, we find a region in which we are unable to continue the model's oscillatory behavior. In this region all trajectories seem to approach the virgin state asymptotically. For the ABG model this region is $3.99 \leq \mu<11$. The left boundary of the region is again 

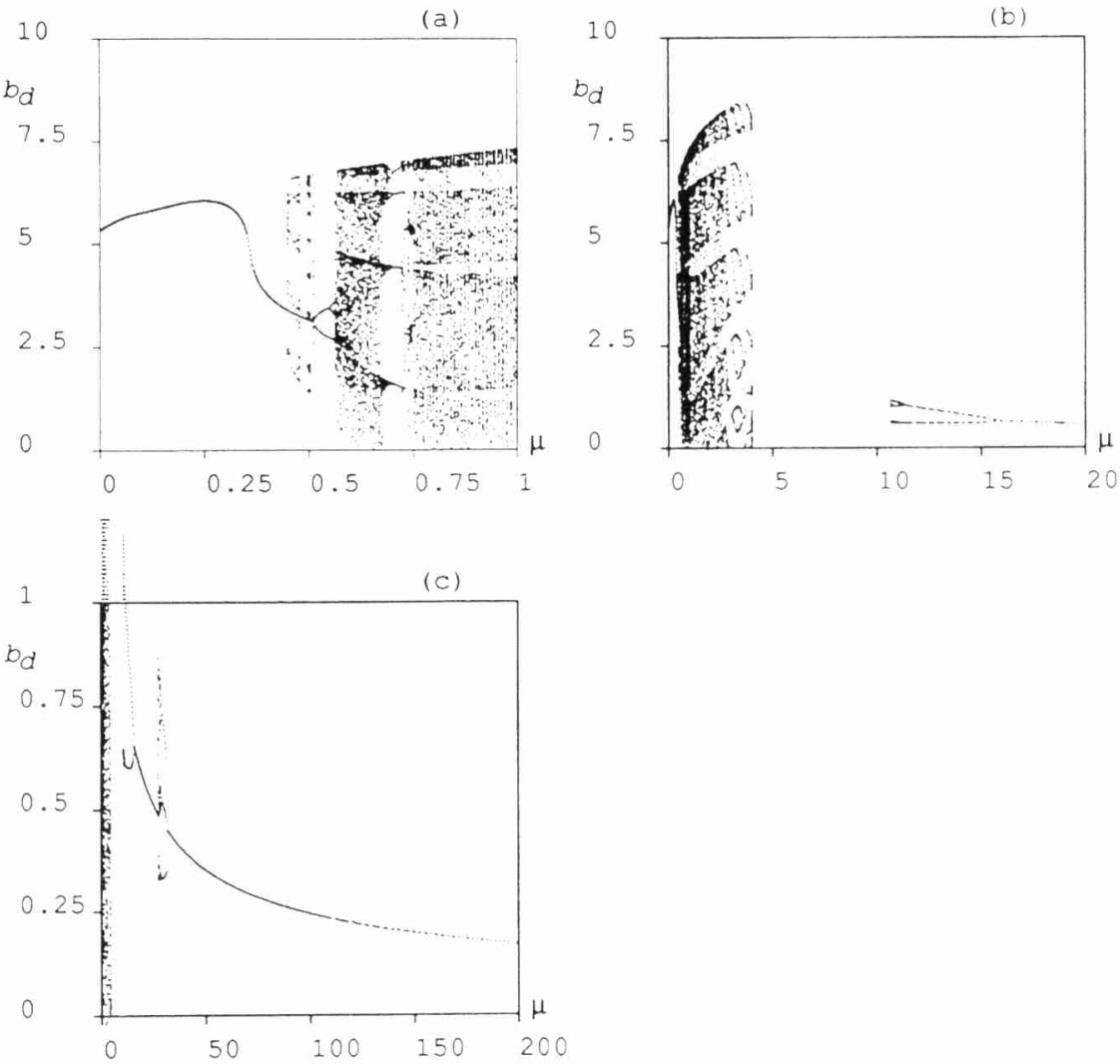

Figure 7. Bifurcation diagram of the gearing up $\mathrm{AB}$ model with respect to the parameter $\mu$. For each value of $\mu$ we plot $20 b$ values attained at a Poincare section at $a_{\mathrm{d}}=0$. Parameters: $\delta=0.1, \eta=0.4, \theta=10 . \rho=2$ and $\sigma=1.48 \times 10^{-3}$. (a) $0<\mu \leqslant 1$; (b) $0<\mu \leqslant 20$; (c) $0 \leqslant \mu \leqslant 200$

related to global bifurcations involving the stable manifold of the MM state. We studied the outsets of the $\mathrm{HH}$ state and the asymmetric $\mathrm{HM}$ and $\mathrm{MH}$ states for an indication of bifurcations involving the inset of the MM state. Although the bifurcation diagrams of the basic $\mathrm{AB}$ model (Fig. 2) and the $\mathrm{ABG}$ model (Fig. 7) look similar, the global bifurcations in which the unstable manifolds of the $\mathrm{HH}$ state and of the asymmetric states are involved are different. For $3.99<\mu<11.5$ these unstable manifolds apparently have a common fate in the $\mathrm{ABG}$ model. For each value of $\mu$, the three different unstable manifolds asymptotically approach the same attractor (i.e. a steady state, a limit cycle or an apparently chaotic attractor). Thus, when one of them is involved in a global bifurcation, so apparently are the others. Since at the bifurcation point $\mu \approx 3.99$ all three unstable manifolds asymptotically approach the virgin state, it seems 
likely that the large amplitude attractor itself disappears here. (Note that in the $\mathrm{AB}$ model the attractor persists in this parameter range and that the unstable manifolds of the $\mathrm{HH}$ and the asymmetric $\mathrm{HM}$ and $\mathrm{MH}$ states are involved in different bifurcations at different values of $\mu$.)

Thus, for $\mu \leqslant 3.98$ the one-dimensional unstable manifold of the HH state asymptotically approaches an attractor on which there is large amplitude timedependent behavior. For $\mu>3.98$ it asymptotically approaches the virgin state (not shown). At the same value of $\mu$ we also found a bifurcation involving the two-dimensional unstable manifold of the asymmetric states: for $\mu \leqslant 3.98$ all trajectories on this hyperplane approach the large amplitude time-dependent behavior. For $3.99<\mu<11.5$ all trajectories on the unstable manifolds of the $\mathrm{HM}$ and MH states seem to approach the virgin state. Since the unstable manifold of the HH state first spirals around the MM state for $\mu \approx 3.98$, we expect a heteroclinic connection between the $\mathrm{HH}$ and the MM states close to this value of $\mu$. Something similar is probably true for the unstable manifold of the asymmetric states (cf. Fig. 3c).

Around $\mu \simeq 11.5$ the structure of the unstable manifolds of the $\mathrm{HM}$ and $\mathrm{MH}$ states becomes similar to the structure described in the AB model: some trajectories on it will approach the virgin $\mathrm{ZZ}$ state, while other very nearby trajectories will asymptotically approach a chaotic attractor (or stable limit cycles in its periodic windows). Thus, at this bifurcation point the apparently chaotic attractor is formed again. Since the formation of the attractor probably also involves the stable manifold of the MM state, we expect another global bifurcation (i.e. a heteroclinic connection) between the unstable manifolds of $\mathrm{MH}$ and $\mathrm{HM}$ states and the stable manifold of the MM state around $\mu \simeq 11.5$. Beyond this bifurcation point we are again able to continue the attractor by our zero-order continuation (see Fig. 7).

Apart from the difference in the unstable manifolds of the $\mathrm{HH}$ and the $\mathrm{MH}$ and $\mathrm{HM}$ states, an important difference between the gearing up $\mathrm{AB}$ model (for $\gamma=0.4$ ) and the basic AB model (for $\gamma \rightarrow x$ ) is the absence of a Hopf bifurcation in the $\mathrm{ABG}$ model. For our parameters the immune states are not stable in the $\mathrm{ABG}$ model. In the parameter range where the immune state was stable in the basic $\mathrm{AB}$ model without gearing up (i.e. for $\mu>12.6$ ) we now find limit cycles, see Figs $7 b$ and $c$. In this region the limit cycles are stable but are difficult to attain (see below). In the region $10 \leqslant \mu \leqslant 20$ we observe a symmetry breaking bifurcation around $\mu=15.7$ (see Fig. 7b) and a cascade of period doublings around $\mu=11.3$ and $\mu=10.9$. where the behavior becomes chaotic. We seem to lose the chaotic attractor just after the Feigenbaum cascade. In the region $20 \leqslant \mu \leqslant 200$ the behavior is a symmetric limit cycle with a small window of period doublings around $\mu=30$, see Fig. $7 \mathrm{c}$.

Similar to that described for the basic AB model, the limit cycles are robust to setting $\sigma=0$ once the attractor has reappeared, i.e. for $\mu \geqslant 11.5$. Other points of 
immunological interest are that the period of the limit cycle decreases if we increase $\mu$ (not shown) and that the oscillations decrease in amplitude (see Fig. 7c). For high values of complex elimination around our estimate $\mu=200$, the limit cycle has a period of 6 days. The 2-week periods reported by Lundkvist et al. (1989) and Varela et al. (1991) are obtained when $\mu \approx 40$. However, because the limit cycles are found beyond the heteroclinic connection between the $\mathrm{HH}$ and the MM state, the behavior is difficult to attain starting from realistic initial conditions (see below) and is not expected to be observed frequently.

\section{The CABG Model-A Two-compartment AB Model with Gearing Up. In} this section we study the full eight-equation CABG model developed in Part I, in which we assume that all of the B cells of interest are localized in the spleen and that some of the antibodies secreted by these cells enter the bloodstream where they can form complexes. Because the antibody circulates, it can also reenter the spleen and effect the local antibody concentrations in that organ. To study the CABG model we set $\gamma=0.4$, our default value for the gearing-up process, we assume $i>0$ and study the behavior of the model as a function of $\lambda$ (the dimensionless inverse residence time for antibodies in the spleen).

\subsection{Stability of the immune states. For $\delta=0.1, \mu=10$ and continuing $i$ as a} bifurcation parameter we find two Hopf bifurcations of the immune states, i.e. a "low Hopf" at $\lambda=0.8$ and a "high Hopf" at $i=249$. In Fig. 8a we continue the two Hopf bifurcations in a two-parameter diagram, varying $\mu$ and $\lambda$ simultaneously. The two Hopf bifurcations enclose an area that we have shaded to indicate the stability of the immune states. For low values of $\lambda$, i.e. for a long residence time in the spleen, this model reduces to the one-compartment model analysed in Fig. 7 . In this region, i.e. $\lambda \ll 1$, we again observe that for $\gamma=0.4$ and any value of the rate of complex turnover, $\mu$, the immune states are unstable. However, for larger values of $\lambda$ the immune states are stable for a large range of values of the residence time in the spleen. Although the stable region is determined by both $\mu$ and $i$, the effect of $i$ is clearly stronger. This suggests that the crucial time-scale determining the stability of the immune states is the residence time in the spleen, $i$. Similar conclusions have been reached before with a two-compartment $\mathrm{AB}$ model lacking gearing up (Perelson and Weisbuch, 1992).

The residence time $\tau$ of the antibody in the spleen is inversely related to i., i.e. $i=\left(\tau d_{\mathrm{B}}\right)^{-1}$, where $d_{\mathrm{B}}$ is the rate of $\mathrm{B}$ cell turnover (see Part I). Thus, the results of Fig. 8 a can be roughly summarized by stating that the immune states are unstable when the residence time is short, i.e. $i>100$ or $\tau<0.5 \mathrm{hr}$, and when the residence time is long, i.e. $i<1$ or $\tau>2$ days. Using the subscripts $\mathrm{S}, \mathrm{M}$ and $\mathrm{L}$ to denote short, moderate and long, we have three categories of residence 

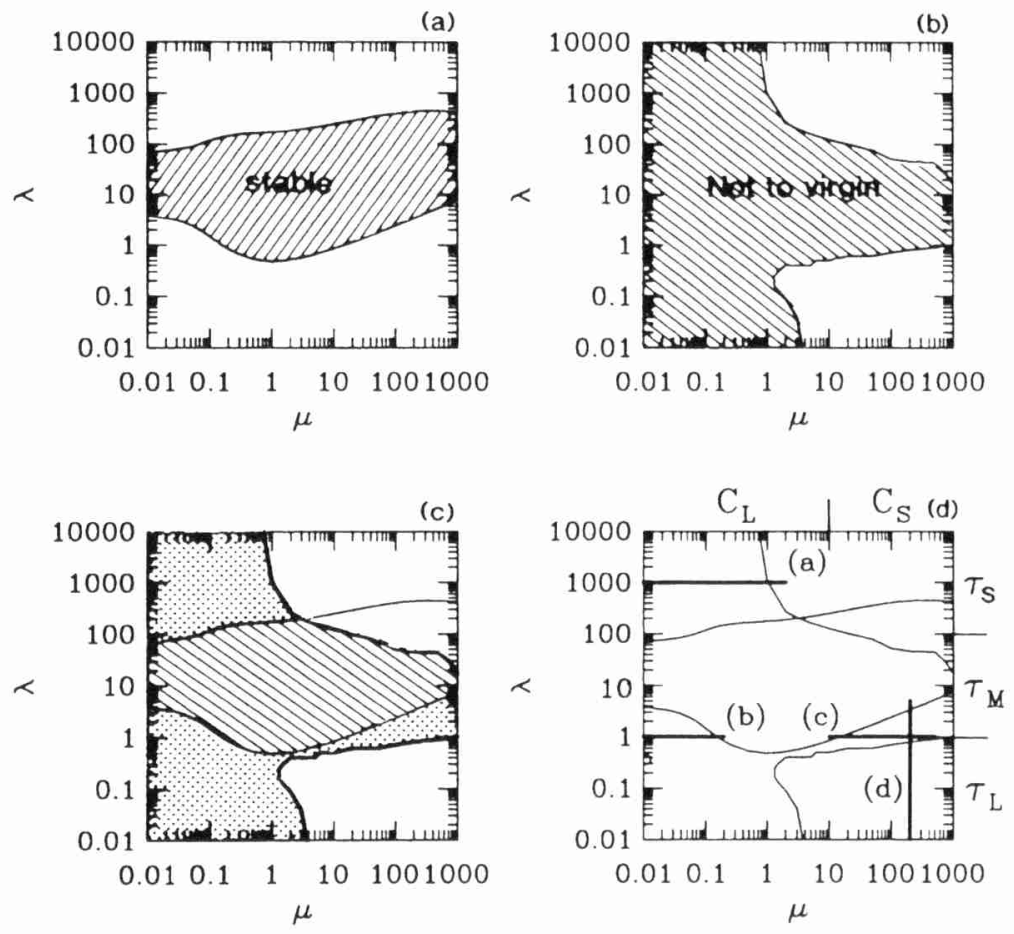

Figure 8. The CABG model. Two parameter bifurcation diagrams, varying $\mu$ and as bifurcation parameters. Parameters: $\delta=0.1, \theta=10, \rho=2, \sigma=1.48 \times 10^{-3}, \gamma=0.4$ and $v=10^{-3}$. In panel (a) we show a continuation of the two Hopf bifurcations. We have shaded the region in which the immune states are stable. In panel (b) we indicate the approximate location of the heteroclinic connection between the $\mathrm{HH}$ and MM states (involving the unstable manifold of the former and the stable manifold of the latter). We have shaded the region in which the unstable manifold of the $\mathrm{HH}$ state does not approach the virgin state. Instead. it may approach one of the stable immune states, a limit cycle or a chaotic attractor. In panel (c) the lines of panels (a.b) are combined to show the region of attainable stable states (shaded by stripes) and the regions of attainable oscillatory chaotic behavior (shaded by a dotted pattern). Panel (d) is similar to (c) but shows, in heavy lines, the four sections through parameter space depicted in Fig. 9 . The $C$ and $\tau$ symbols indicate the parameter regimes explained in the text.

times, $\tau_{S}, \tau_{M}$ and $\tau_{L}$, for which we find different behavior. Thus, stable immune states are found for moderate residence times, i.e. for $0.5 \mathrm{hr}<\tau_{\mathrm{M}}<2$ days. The residence time of the $\operatorname{IgM}$ antibody in the spleen has not been measured and is difficult to estimate. Whether or not the immune states are biologically expected to be stable therefore remains undetermined.

6.2. Attainability: The stability of activated states is not the only issue of immunological importance. We have shown above, for the basic AB model, 
that stable activated states and or sustained oscillatory or chaotic behavior may have such a small basin of attraction that they are not likely to be attained by stimulating an initially quiescent immune system. We argued above that the basin of attraction of the behavior corresponding to sustained activation is most likely to be related to the high-dimensional stable manifold of the MM state. Further, because of heteroclinic connections between the inset of the MM state and the outsets of the $\mathrm{HH}$ and the $\mathrm{HM}$ and $\mathrm{MH}$ states, these three unstable manifolds may be used as an indicator of changes in the basin of attraction of the sustained behavior. Indeed, we found that the outset of the $\mathrm{HH}$ state provides an indication of the size of the basin of attraction of the sustained oscillatory behavior. Thus, for the CABG model the lines in Fig. 8b depict the global bifurcation in the unstable manifold of the $\mathrm{HH}$ state for $\delta=0.1$. We have shaded the area where this unstable manifold asymptotically approaches the immune states (when they are stable) or when it asymptotically approaches the limit cycle or the chaotic attractor (when the immune states are unstable). In the remaining white area the unstable manifold asymptotically approaches the virgin $\mathrm{ZZ}$ state. The overall picture of the bifurcation involving outset of the HH state is very similar to that shown in Fig. 3. Thus, spiraling in the general neighborhood of the MM state the manifold turns either towards higher values of $a_{\mathrm{s}}$ or towards lower values of $a_{\mathrm{s}}$.

In Fig. $8 \mathrm{c}$ we combine the diagrams of Fig. $8 \mathrm{a}$ and b. Since we only want to consider regions with behavior that is "attainable from reasonable initial conditions", we have only shaded those regions in which the unstable manifold of the $\mathrm{HH}$ state does not approach the virgin state. We use two types of shading to differentiate between the regions in which the attainable behavior is stationary and those in which it is oscillatory chaotic. Thus, with a striped pattern we indicate the region of attainable stationary behavior and with a dotted pattern we denote the regions with attainable oscillatory/chaotic behavior.

The striped area is confined to the $\tau_{M}$ regime. Here. the stability of the CABG model qualitatively corresponds to that of our simplest $\mathrm{B}$ model. This suggests that our previous results on stable immunological memory (De Boer, 1988; De Boer and Hogeweg, 1989b.c; Weisbuch et al., 1990) would be valid if the residence time of the antibody in the spleen falls in this reasonably large region. $0.5 \mathrm{hr}<\tau<2$ days. Note that the limits of this region are determined by both stability and attainability. We will discuss below that even in this "attainable" regime the system need not attain the one steady state that corresponds to immunological memory.

6.3. Behavior in attainable regimes. The dotted areas have a vertical boundary that is located at roughly $\mu=10$. The lifetime $1 / d_{C}$ of complexes is inversely related to $\mu$, i.e. $\mu=v d_{C} d_{B}$ (see Part I). Thus, based upon the lifetime 
of the complexes, we distinguish two parameter regimes. We shall call the lifetime $1 / d_{C}$ of the complexes $C$ and denote the long and short lifetime $C_{\mathrm{L}}$ and $C_{\mathrm{S}}$, respectively. A long lifetime $C_{\mathrm{L}}$ being defined by $\mu=v d_{\mathrm{C}} d_{\mathrm{B}}<10$ or $1 / d_{\mathrm{C}}>$ 2 days and a short lifetime $C_{\mathrm{S}}$ being defined by $\mu=\imath \cdot d_{\mathrm{C}} d_{\mathrm{B}}>10$ or $1 / d_{\mathrm{C}}$ $<2$ days. In the dotted areas of attainable oscillatory chaotic behavior we have performed four Poincare map continuations. The location of these continuations in the parameter space is indicated by the four straight lines in Fig. 8d. The marks (a)-(d) along these lines in Fig. 8 d correspond to the four panels of Fig. 9. The $C$ and $\tau$ symbols in the margin indicate the different parameter regimes.

In the $C_{\mathrm{L}}$ regime, where complexes are long-lived, there are two regions, i.e. $\tau_{\mathrm{S}} C_{\mathrm{L}}$ and $\tau_{\mathrm{L}} C_{\mathrm{L}}$, where the attainable behavior is oscillatory or chaotic. The behavior in the two regions is very different. In Fig. 9a we plot for the $\tau_{\mathrm{S}} C_{\mathrm{L}}$ region $b_{\mathrm{d}}$ values attained at the plane $a_{\mathrm{d}}=0$ for $\lambda=1000$ and several values of $0 \leqslant \mu \leqslant 2$. The behavior resembles that of the basic AB model (Fig. 2) and that of the ABG model for $\mu<10$ (Fig. 7). We see a symmetric cycle going through a symmetry breaking and a period doubling cascade. Then the behaviour becomes chaotic. We lose the chaotic attractor at $\mu \approx 1$, which is around the global bifurcation in the unstable manifold of the HH state (see Fig. 8d at $i=1000$ and $\mu \approx 1$ ).

The behavior in the $\tau_{\mathrm{L}} C_{\mathrm{L}}$ region, e.g. for $\lambda=1$, is very different because we typically find two asymmetric limit cycles winding around one of the immune states (not shown). This behavior is very similar to that shown in Figs $7 \mathrm{e}$ and $7 \mathrm{f}$ of Part I for the parameter region $\mu=0$ and $0.89<\delta<0.98$. In the CABG model the corresponding parameter region is also located at small values of $\mu$ but the region is much larger. It thus appears that for $\mu=0$, setting $i \approx 1$ in the $\mathrm{CABG}$ model has a similar effect to setting $\delta \approx 1$ in the basic AB model. In both cases the lifetimes of the $\mathrm{B}$ cells and the antibodies in the spleen are approximately equal. Since these cycles do not cross the plane $a_{\mathrm{d}}=0$ we show in Fig. $9 \mathrm{~b}$ a continuation of Poincare sections at the plane $a_{\mathrm{s}}=0$. As shown in Fig. 9b, we found limit cycle behavior in the range $0<\mu<0.16$. The upper limit of this range is determined by a supercritical Hopf bifurcation at $i=1$ and $\mu=0.16$ (see Fig. 8d). Here two asymmetric cycles are born. Decreasing $\mu$ increases the amplitude of the two asymmetric limit cycles until they "glue" (see Part I). A symmetric cycle is observed that undergoes a symmetry breaking bifurcation and period doublings. In this $\tau_{\mathrm{L}} C_{\mathrm{L}}$ region the behavior remains periodic.

For short lifetimes of complexes, i.e. in the $\tau_{\mathrm{L}} C_{\mathrm{S}}$ regime, we find the third region of attainable oscillatory/chaotic behavior. This is the narrow zone defined by $i \approx 1$ and $\mu>10$. The straight lines marked by (c) and (d) in Fig. 8d show the location of the two Poincare continuations that we performed in this region. The behavior of the $\mathrm{CABG}$ model in this region resembles the novel behavior obtained with the gearing up model for high values of $\mu$. High values 

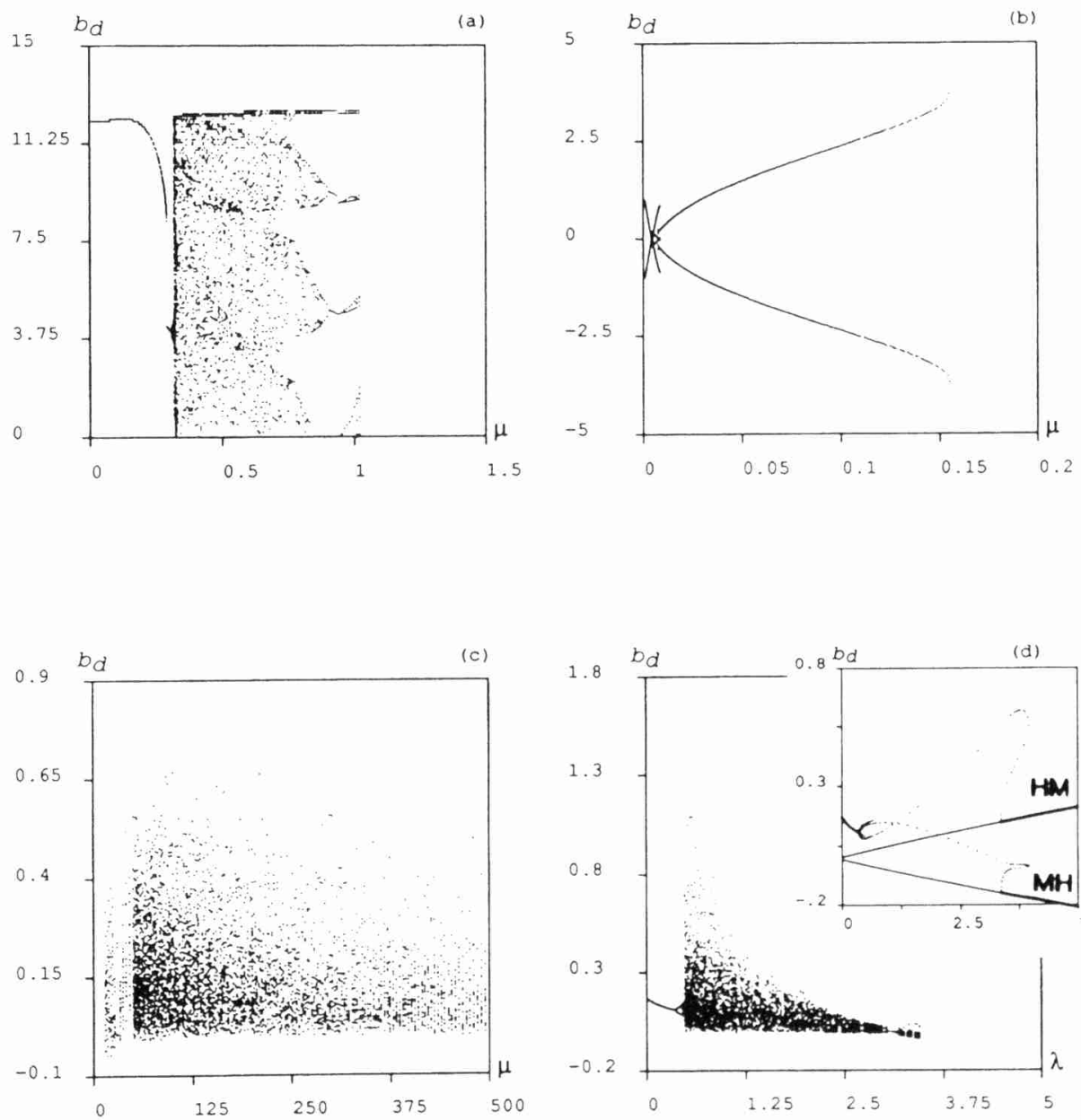

Figure 9 . Bifurcation diagram of the $\mathrm{CABG}$ model varying $\mu$ or $;$ as a bifurcation parameter. For each value of the parameter we plot $20 \mathrm{~b}$ d values attained at a Poincaré section at $a_{\mathrm{d}}=0$ (a), (c) and (d) or $a_{\mathrm{s}}=0$ (b). Parameters: $\theta=10, \rho=2$. $\sigma=1.48 \times 10^{-3} \cdot \gamma=0.4$ and $\gamma=10^{-3}$. (a) For $i=1000$ we vary $0 \leqslant \mu \leqslant 1.5$. We lose the attractor at $\mu=1.02$. which is around the bifurcation involving the unstable manifold of the $\mathrm{HH}$ state at $\mu \approx 1$. (b) For $i=1$ we vary $0 \leqslant \mu \leqslant 0.2$. We find stable limit cycles that are born at the supercritical Hopf bifurcation at $\mu=0.16$. (c) For $i=1$ we vary $0 \leqslant \mu \leqslant 500$. We loose the attractor at $\mu=13.4$ which is beyond the subcritical Hopf bifurcation at $\mu=14.9$. Thus, there is hysteresis for $13.4<\mu<14.9$. (d) For $\mu=200$ we vary $0 \leqslant i \leqslant 5$. We lose the attractor at $\mu=3.5$ which is beyond the subcritical Hopf bifurcation at $i=3.36$. Thus, there is hysteresis for $3.36<i<3.5$. At the bottom end, the attractor persists to $i=0$. In the inset we show one parameter continuations of the immune states and of the limit cycles that are born at the Hopf bifurcations which these states are involved in. Heavy lines and dots denote stable steady states and stable limit cycles, respectively. Each dot represents the maximum value of $b_{d}$ attained during a limit cycle. Light lines and dots denote unstable states and cycles. 
of $\mu$ mean that the antibody is rapidly eliminated by the formation of complexes. This has a number of effects. First, the shape of the chaotic attractor and the limit cycles in its periodic windows is different. The attractors are very flat in the $a_{\mathrm{s}}=\ln A_{1}+\ln A_{2}$ direction. This means that the antibody concentrations are strongly correlated, i.e. any rise in $A_{1}$ is counterbalanced by an immediate decline in $A_{2}$. Additionally, the $\mathrm{B}$ cells and the gearing up functions hardly change in time and remain near the equilibrium value of the immune states (not shown). Second, in the antibody phase space the cycles do not wind around the immune states but tend to remain below the line $a_{\mathrm{s}}=0$ (see Fig. 10). Third, for $\mu=200$ typical periods of limit cycles in the CABG model are of the order a few days to a week. Thus, the oscillatory chaotic behavior has a short time scale in the $C_{\mathrm{S}}$ regime. We will discuss the immunological implications of this in the section on experimental data.

The two Poincare continuations in the $\tau_{\mathrm{L}} C_{\mathrm{S}}$ regime are shown in Figs $9 \mathrm{c}$ and $\mathrm{d}$ (plotting $b_{\mathrm{d}}$ values attained at $a_{\mathrm{d}}=0$ planes). In Fig. $9 \mathrm{c}$, for $i=1,0 \leqslant \mu \leqslant 500$, we see a large region of chaotic behavior in which the variation in $b_{\mathrm{d}}$ is small, i.e. $-0.1<b_{\mathrm{d}}<1$. This means that the amplitude of the $\mathrm{B}$ cell populations is very small. In Fig. 9 d. for $0 \leqslant i \leqslant 5, \mu=200$, we see a symmetry breaking (at $i \approx 0.367$ ) and a period doubling cascade (starting at $i \approx 0.428$ ) that evolves into chaos. We loose the attractor around $\lambda=3.5$. which is just above the Hopf bifurcation at $i=3.36$. Thus, we have a small region of hysteresis where the chaotic attractor co-exists with stable immune states and a stable virgin state (cf. Fig. 4f).

We have studied the death of the attractor around $i=3.5$ by continuing with AUTO (Doedel. 1981), the unstable limit cycles that are born at the subcritical Hopf bifurcations in the immune states at $\lambda=3.36$ (see the inset of Fig. 9d). In this diagram the two straight lines form the one parameter continuations of the HM and MH states. The Hopf bifurcations correspond to the points where these lines switch stability. In the inset heavy lines and dots denote stable steady states and stable limit cycles, respectively. (In fact, the heavy dots in the regions $0<\mu<0.43$ are so fat that they appear as a heavy line.) Each dot represents the maximum value of $b_{\mathrm{d}}$ attained during a limit cycle. Light lines and dots denote unstable states and cycles.

Thus, the light dots that appear above the two Hopf bifurcations denote the two unstable limit cycles that are born there. The asymmetric cycles increase in amplitude with increasing $i$ until a turning point bifurcation at $i \approx 3.95$. Beyond this turning point. i.e. for lower values of $i$, the asymmetric cycles remain unstable until a bifurcation point at $i \approx 0.428$. The latter bifurcation is the period doubling that we also found in the Poincare sections of Fig. 9d. We have also continued the stable limit cycle branch of the period doubling bifurcation (see the inset). This branch rapidly becomes unstable due to another period doubling. We have seen above that this leads to chaos. 
(a)

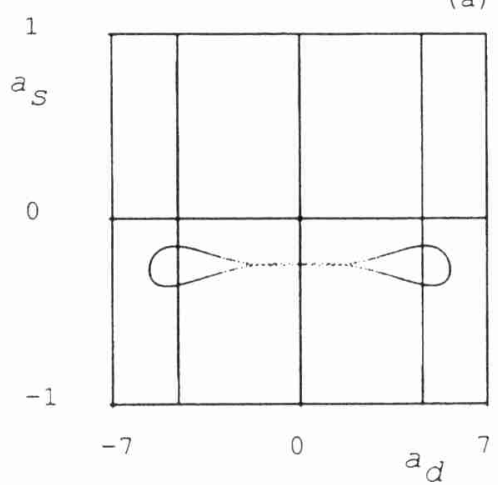

(c)

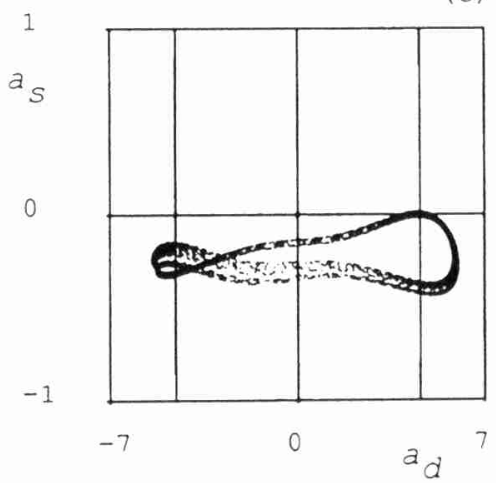

(b)

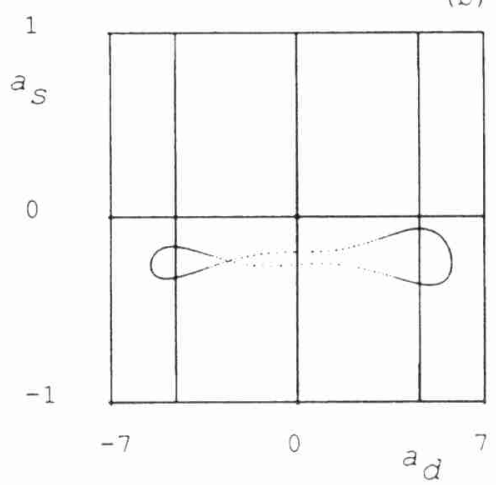

(d)

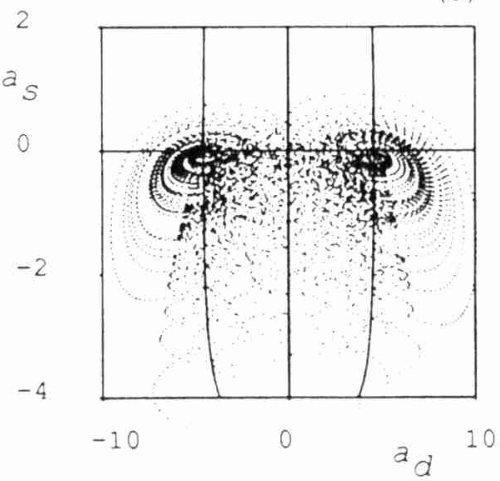

Figure 10. Symmetry breaking. period doublings and chaotic behavior in the CABG model. Parameters: $\theta=10, \rho=2 . \sigma=1.48 \times 10^{-3} \cdot \delta=0.1, \mu=200, \gamma=0.4$ and $v=10^{-3}$. (a) $i=0.3$ : symmetric limit cycle (period 8.4 days). (b) $i=0.38$ : asymmetric limit cycle (period 8.6 days). (c) $i=0.45$ : noisy periodicity. (d) $i=0.5$ :

(apparent) chaos.

Continuing the asymmetric limit cycles beyond the first period doubling bifurcation, i.e. towards smaller values of $i$, the two asymmetric cycles merge at the pitch-fork (symmetry breaking) bifurcation of $i \approx 0.367$. The stable branch of the symmetric limit cycle of the pitch-fork bifurcation is continued towards $i \approx 0$. The unstable branch is continued for a few steps, as is indicated in the inset by a few light dots.

The unstable limit cycles that are born at the two Hopf bifurcations, along with their stable manifolds probably form the basin boundary of the apparently chaotic attractor. We lose the attractor around $i \approx 3.5$. This may again be related to a global interaction involving the stable and unstable manifolds of the growing unstable saddle-type limit cycles. Although we enter the region 
where the outset of the HH state asymptotically approaches the virgin state at $i \approx 0.8$ we are able to continue the attractor in this region.

Specific examples of the flat limit cycles in the $\tau_{L} C_{S}$ regime (here for $\mu=200$ ) are shown in Fig. 10. A symmetric limit cycle from the $i<0.367$ regime is shown in Fig. 10a. One of the asymmetric cycles that are born at the (pitchfork) symmetry breaking bifurcation around $i=0.367$ is shown in Fig. 10 b. The two asymmetric cycles remain below both immune states. Following the period doubling around $i=0.43$ they develop noisy periodicity around $i=0.45$ (Fig. 10c). Full blown chaos is observed for $i \geqslant 0.48$ (Figs 9d and 10d).

Finally, we have checked the $\tau_{S} C_{S}$ regime in which the unstable manifold of the $\mathrm{HH}$ state asymptotically approaches the virgin state. (See the non-shaded area in the top right-hand corner of Fig. 8c.) In this region we find long transients that are seemingly chaotic. However, all trajectories that we have computed eventually approach the virgin $\mathrm{ZZ}$ state.

6.4. Antigens and memory. Studying basins of attraction is even harder in the CABG model than it is in the basic AB model. As before, we restrict our attention to studying where typical initial conditions involving stimulation with an antigen will ultimately go. We now consider an antigen. $P$, that circulates between the periphery and the spleen. Similar to the notation for antibodies, we let $\hat{P}$ be the peripheral antigen concentration and $P$ be the antigen concentration in the spleen. In the two compartment model $P$ is recognized by $B_{1}$ cells and the $A_{1}$ antibody, whereas $\hat{P}$ is recognized by the $\hat{A}_{1}$ antibody. The antigen is removed from the system by the formation of antigen-antibody complexes in the spleen and in the periphery. For the activation of the $B_{1}$ cells we use equations (11) and (12) and the gearing up equation:

$$
\frac{\mathrm{d} G_{1}}{\mathrm{~d} T}=\eta\left(f(h)-G_{1}\right),
$$

where $h=A_{2}+P$. The antibody equations now both contain a new term corresponding to the loss of antigen-antibody complexes, i.e.:

$$
\begin{gathered}
\frac{\mathrm{d} A_{1}}{\mathrm{~d} T}=\delta\left(B_{1} G_{1}-A_{1}\right)-i\left(A_{1}-\hat{A}_{1}\right)-\mu A_{1} A_{2}-\kappa A_{1} P \\
\frac{\mathrm{d} \hat{A}_{1}}{\mathrm{~d} T}=v\left(A_{1}-\hat{A}_{1}\right)-\delta \hat{A}_{1}-\mu \hat{A}_{1} \hat{A}_{2}-\kappa \hat{A}_{1} \hat{P}
\end{gathered}
$$

where $k$ is the rate at which antigen-antibody complexes are removed. The antigen, assumed to be non-growing, is governed by the equations: 


$$
\begin{gathered}
\frac{\mathrm{d} P}{\mathrm{~d} T}=-\delta_{P} P-\lambda(P-\hat{P})-\kappa A_{1} P \\
\frac{\mathrm{d} \hat{P}}{\mathrm{~d} T}=-\delta_{P} \hat{P}+\lambda_{(}(P-\hat{P})-\kappa \hat{A}_{1} \hat{P}
\end{gathered}
$$

where $\delta_{p}$ is the rate of antigen loss due to non-specific removal mechanisms. For $\delta_{P}>0$, the long-term behavior does not involve the antigen (since it asymptotically disappears by turnover) and therefore ultimately the dynamics, and the stability properties of steady states, are the same as before. Thus, the antigen only affects where a particular trajectory will go. We introduce the antigen in the periphery at a maximally stimulatory dose, i.e. $\hat{P}(0)=1, P(0)=0$, with the system in the virgin ZZ state, i.e. $B_{1}=B_{2}=\sigma$ and $A_{1}=A_{2}=\hat{A}_{1}=\hat{A}_{2}=$ 0 . This activates the system and we ask whether or not the system remains activated after the antigen is removed.

The model is studied for various values of $k, \mu$ and $;$ in Fig. 11 . We have shaded the region where the system remains activated after stimulation, i.e. where it does not return to the virgin state following the elimination of the antigen. We discretize parameter space by sampling the behavior for $\mu=10^{-2}$, $10^{-1.5}, 10^{-1}, \ldots, 10^{1.5}, 10^{2}$ and for $i=10^{-2} \cdot 10^{-1.5}, 10^{-1} \ldots \ldots 10^{3.5} \cdot 10^{4}$. We first repeat the situation without the antigen. Thus. Fig. 11 a is a discretized version of Fig. 8b, where we have shaded the region in which the unstable manifold of the $\mathrm{HH}$ state does not approach the virgin state. In Figs $11 \mathrm{~b}$ d, for three values of $k$, we have shaded the regions where the system does not return to the virgin state following the elimination of the antigen. The similarity between Fig. 11 a and Figs $11 \mathrm{~b}-\mathrm{d}$ suggests that the unstable manifold of the $\mathrm{HH}$ state is related to the attainability of sustained behavior (following stimulation with the antigen). Thus, the asymptotic fate of the unstable manifold of the $\mathrm{HH}$ state seems to be the same as that of the network stimulated with the antigen. The shaded regions of Fig. 11b-d are largely contained in the region shaded in Fig. $11 \mathrm{a}$ (except for $i=10^{-0.5} \simeq 0.3$ and $\mu=10^{0.5} \simeq 3$ ). Our simulations, therefore, suggest that the $\mathrm{CABG}$ model is only expected to attain sustained activation in the parameter region where the outset of the $\mathrm{HH}$ state does not approach the virgin state. Above, we reached the same conclusion for the basic $\mathrm{AB}$ model.

Depending on the location in the ivs $\mu$ parameter space, the behavior that is attained in Fig. 11 is either (1) a stationary state in the $\tau_{M}$ regime, (2) a cycle around one immune state in the $\tau_{\mathrm{L}} C_{\mathrm{L}}$ regime, (3) an oscillation around both immune states or (4) chaos in the $\tau_{\mathrm{S}} C_{\mathrm{L}}$ and $\tau_{\mathrm{L}} C_{\mathrm{S}}$ regimes. Behavior (1) and (2) may account for a type of immunological memory, because the system switches from the virgin state to a state in which one of the clones is kept immune. We 

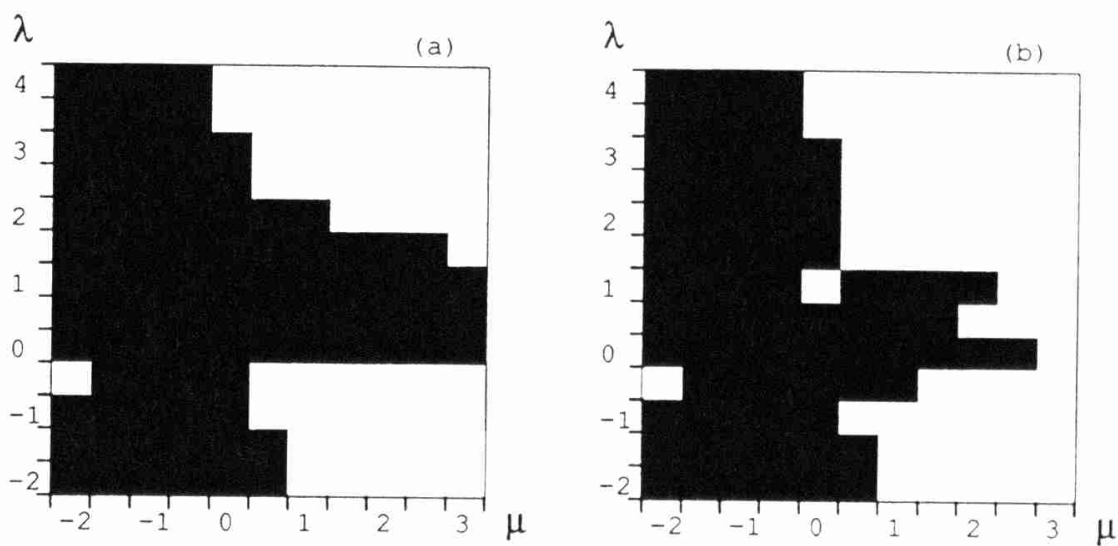

$\lambda$

(c)

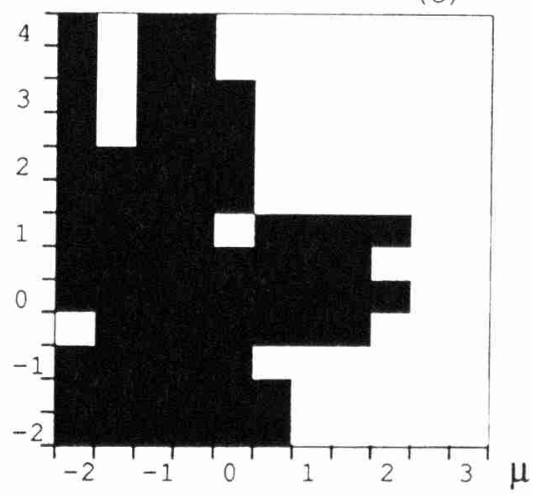

$\lambda$

(d)

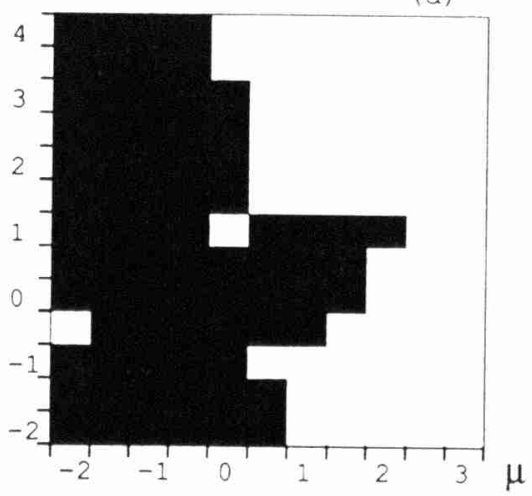

Figure 11. Attainability of sustained behavior of the CABG model after antigen stimulation. As a function of $\mu$ and $i$ [on a logarithmic scale (base 10)] we shade the region in which the system does not ultimately approach the virgin state. Instead. it may approach one of the activated states of the system. be it either a stable immune state, a stable limit cycle or a chaotic attractor. Parameters: $\theta=10, \rho=2$. $\sigma=1.48 \times 10^{-3} \cdot \gamma=0.4$ and $r=10^{-3}$. In panel (a) no antigen stimulation was included and we show the ultimate fate of the unstable manifold of the $\mathrm{HH}$ state (this is the same as the shaded region in Fig. 8b). In panels (b-d) we introduce the antigen. $\hat{P}_{(0)}=1$. in the virgin $\mathrm{ZZ}$ state for (b) $k=10^{-2}$. (c) $k=10^{-3}$ and (d) $\kappa=10^{-4}$.

refer to our previous work for a more detailed description of this form of idiotypic memory (De Boer. 1988: De Boer and Hogeweg, 1989b; Weisbuch et al., 1990). However, closer inspection of the behavior attained in Fig. 11 reveals that the immune state that is attained following the elimination of the antigen need not be the correct one. Thus. following the stimulation of clone 1 with the antigen $P$ we frequently attain the $\mathrm{MH}$ state where clone 1 is suppressed and clone 2 is immune. This is due to transient fluctuations (i.e. 
"pre-chaotic" transients) during the immune response to the antigen that bring the system in the "wrong" basin of attraction. The attainment of different attractors has also been described for the B model (Weisbuch et al., 1990) and has been explained analytically in terms of parameter conditions (Neumann and Weisbuch, 1991). For the CABG model determining which attractors are attained is much more complicated and requires new studies.

For behavior (3) and (4) the system as a whole remains activated but each clone alternates from a suppressed to an immune state. Such an activated state can account for memory but no longer distinguishes between tolerance and immunity. Finally, note that for $\mu>316$ stimulation with the antigen never gives rise to sustained activation. Thus, whenever complexes are rapidly removed, the network does not easily account for memory.

6.5. Comparison to experimental data. The natural serum $\operatorname{Ig} M$ antibody levels of idiotypic and anti-idiotypic antibodies have been measured in unimmunized mice (Lundkvist et al., 1989; Varela et al., 1991). In order to obtain time series data the mice were bled periodically and antibody concentrations in the sample measured. (Repeated measurements of antibody concentrations in the spleen are much more difficult.) Since absolute concentrations are difficult to be determined. Lundkvist et al. report data in which the concentration at day zero is arbitrarily set to $100 \%$. The concentrations in all subsequent samples are scaled accordingly.

The data showed that idiotypic antibody concentrations fluctuate on a short time scale (i.e. of the order 2 weeks) and that the amplitude of the fluctuations was small (i.e. two- to three-fold). Looking at the data reported by Lundkvist et al. (1989) for correlations in the concentration changes of idiotypic-antiidiotypic pairs, we have been able to show (unpublished result) that the increase in concentration of one antibody correlates with the decrease of the anti-idiotypic antibody $\left(x^{2}=7.2, p<0.01, n=36\right.$, i.e. six mice with six weekly samples). Although there is too little data to be conclusive, Lundkvist et al. (1989) and Varela et al. (1991) suggest that the concentration fluctuations are compatible with the existence of chaotic dynamical behavior. Treatment of the mice with very low doses of either idiotypic or anti-idiotypic antibodies resulted in the inhibition of the fluctuations for months.

The behavior of our model seems to be in general accordance with these experimental results. First, our model gives oscillatory and chaotic behavior. Second, the model is indeed capable of predicting a period of two cycles per month and an amplitude of two- to three-fold variations in peripheral antibody concentrations. Third, one might speculate that the small basins of attraction explain why one can inhibit the oscillatory behavior with small doses of antiidiotypic antibodies.

In order to test this correspondence between experimental and theoretical 
results more rigorously we performed similar experiments with the $\mathrm{CABG}$ model in the chaotic $\tau_{\mathrm{L}} C_{\mathrm{S}}$ regime, i.e. $\lambda=1$ and $\mu=200$ (Fig. 12). In panels (a) and (b) of Fig. 12 we show a time series of the peripheral antibody concentrations $\hat{a}_{1}$ and $\hat{a}_{2}$ in $\mu \mathrm{g} / \mathrm{ml}$ on a logarithmic (Fig. 12a) and a linear scale (Fig. 12b). First, the concentrations that we observe are of the order $1 \mu \mathrm{g} / \mathrm{ml}$. Since total serum IgM concentrations are typically of the order $1 \mathrm{mg} / \mathrm{ml}$ (Eisen. 1980), the concentration of each idiotype seems to be quite large. We argued in the discussion of Part I that such high concentrations may be a consequence of our parameter choices. Second, we observe small short-term fluctuations on a time scale of a few days, and large long-term oscillations on a time scale of weeks. Third, the amplitude of the oscillations in the linear plot seems roughly five-fold around an average value of $1 \mu \mathrm{g} / \mathrm{ml}$. Both the amplitude and frequency of the oscillations agree with the experimental data (Lundkvist et al.. 1989: Varela et al.. 1991).

Finally, we simulated the experiment performed by Lundkvist et al. (1989) in which a small amount of one of the two antibodies is introduced. In Figs 12c and $\mathrm{d}$ we show the effect of introducing $10 \mu \mathrm{g}$ of anti-idiotypic antibody at day zero. (Lower doses tend to have no effect in the model.) Otherwise, the initial conditions are identical to those in panels (a) and (c). The linear plot in Fig. 12d shows that such a perturbation of the system would manifest itself in experimental measurements as the absence of anti-idiotypic fluctuations for about 50 days and as a slowly declining concentration of the anti-idiotypic antibody that was administered at day zero. The experimental data are in agreement with the former observation, but at variance with the latter.

7. Discussion. In this paper we have studied the dynamics and bifurcation behavior of three different $\mathrm{AB}$ models: the basic AB model with antibodies and $\mathrm{B}$ cells, the gearing up or ABG model in which a delay in the antibody secretion due to the $\mathrm{B}$ cell maturation is included and finally the CABG model in which spleen and blood compartments are distinguished. The typical behavior of the basic AB model is the attainment of a stationary state, or oscillations and chaos on a time scale of several months. We have suggested that the stationary states are unlikely to be attained because they have a small basin of attraction. In the gearing up $\mathrm{AB}$ model the immune states are not stable for the parameter regimes we believe to be realistic and the typical behavior of the model is oscillatory, either limit cycles or chaos. The time-scale of the oscillations is fast when the lifetime of the complexes is less than 1 day. However, in this short complex lifetime $C_{\mathrm{S}}$ regime the limit cycles seem to have a small basin of attraction and are unlikely to be attained.

The CABG model has a variety of types of different behavior for different parameter regimes. The immune states are stable and attainable for a large range of antibody residence times, $\tau$, in the spleen, i.e. for $0.5 \mathrm{hr}<\tau<2$ days. 

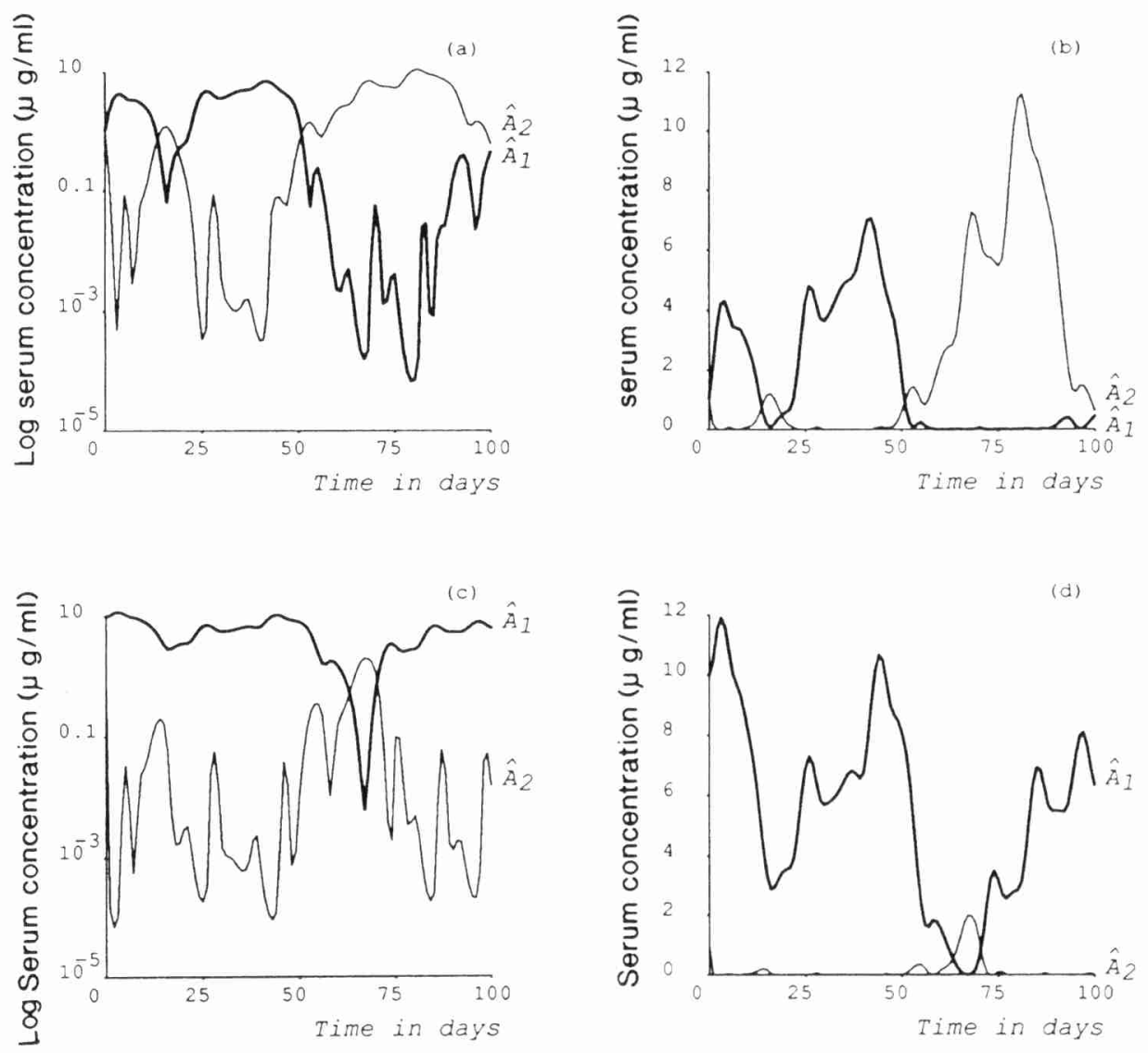

Figure 12. Peripheral antibody concentrations in the CABG model. Parameters: $\theta=10, \rho=2 . \sigma=1.48 \times 10^{-3} \cdot \delta=0.1, \mu=200 . i=1, \gamma=0.4$ and $\gamma=10^{-3}$. (a) $\hat{a}_{1}$ and $\dot{a}_{2}$ on a logarithmic scale. (b) $\dot{a}_{1}$ and $\dot{a}_{2}$ on a linear scale. Notice that the concentrations vary roughly by a factor of five. (c) The effect of introducing $\hat{a}=\left.10 \mu \mathrm{g} \mathrm{ml}\right|^{-1}$ at day zero on a logarithmic scale. (d) The effect of introducing $\dot{a}_{2}=10 \mu \mathrm{g} \mathrm{ml}$ at day zero on a linear scale.

Outside this region we find oscillations. We have defined $\tau_{S}$ and $\tau_{L}$ as the regimes in which $\tau<0.5 \mathrm{hr}$ and $\tau>2$ days, respectively, and $C_{\mathrm{S}}$ and $C_{\mathrm{L}}$ as the regimes in which the lifetime of complexes $1 d_{C}$ is $<2$ days and $>2$ days, respectively. Thus, we find attainable oscillatory chaotic behavior with a time scale of several months in the $\tau_{\mathrm{S}} C_{\mathrm{L}}$ regime. We find attainable oscillations around one immune state, which was typical of our simplest B model, in the $\tau_{\mathrm{L}} C_{\mathrm{L}}$ regime. Finally, in the $\tau_{\mathrm{L}} C_{\mathrm{S}}$ regime we find attainable oscillations and chaos on a time scale of days to weeks. 
Unfortunately, the residence time of the antibody in the spleen and the lifetime of idiotypic complexes, i.e. our parameters $i$ and $\mu$, which are so crucial for the model behavior, are very difficult to measure experimentally. Our original estimates of the two parameters may be inaccurate. Fitting the theoretical results obtained in this paper to the limited experimental data (Lundkvist et al.. 1989: Varela et al.. 1991) may give us some clues as to the parameter values. Since these data suggest oscillations with a time scale of weeks, our theory would predict that $\mu$ should be of the order 100 . The lifetime of the complexes would then be of the order $5 \mathrm{hr}$, which is quite feasible. Speculating even further, we could argue that since the oscillatory chaotic behavior was found (and re-established) experimentally, the attractor should be attainable. Combining this with the constraint that the immune states are unstable, we speculate that this corresponds to the $\tau_{L}$ regime. Thus, the average residence time of antibodies in the spleen. $\tau$, should be of the order 1-2 days. This is longer than the suggested residence time for cells brought into the spleen (Sprent, 1989).

The speculation that the empirical data correspond to our $\tau_{\mathrm{L}} C_{\mathrm{S}}$ regime should be taken with extreme care because the CABG model still contains enormous simplifications. One major concern is the fact that we study the interaction of only one idiotype with only one anti-idiotype. The immune network with which the experimental data are obtained probably consists of thousands to millions of connected idiotypes. The loss of stability of such a system may be a distributed property of the network due to interaction between many populations (Gardner and Ashby, 1970: May, 1972: De Boer and Hogeweg, 1989a) and need not be related to a local Hopf bifurcation of an immune state.

Our models are also unrealistic in other regards that may effect the stability of the immune states. The symmetric activation function that we have used is characteristic of bivalent antibodies (Perelson and DeLisi, 1980). Crosslinking of receptors by 10 -valent IgM can give rise to crosslinking curves that are not symmetric (Perelson, 1981). An improvement of our models would be to better account for the chemistry involved in IgM binding and crosslinking. Also, it is clear that antibody-antibody complexes that form in solution may be of different sizes. This is an additional complication that we have yet to consider. Lastly, we have not explicitly considered the roles of antigen processing, antigen presentation and $\mathrm{T}$ cells.

The symmetric logarithmic (SL) coordinate system that we have employed here has proved useful for defining Poincare sections at the plane of symmetry. A comparison of the trajectories plotted in the original and in the SL coordinate system (e.g. Fig. 3) shows that this difference appears as merely a rotation of the state space. Conversely, we observed in Part I that the interpretation of the nullclines of the SL coordinate system is not intuitive. 
Since the SL coordinate system has not revealed any new insights, it might have been better to present all figures in the original coordinate system. Thus, we could have computed the Poincare sections in the SL coordinate system, but represented their results in the original coordinate system.

One of the most interesting new results found for the CABG model is that for high rates of complex removal. $\mu$, sustained oscillatory chaotic behavior persists, even if $\sigma$, the source of cells from the bone marrow, is zero. This may be particularly important for models in which instead of a continuous source of cells of established clones there is a stochastic source of novel clones (De Boer and Perelson, 1991). Stewart and Varela $(1989,1990)$ have also searched for conditions for which oscillatory chaotic behavior could be sustained in the absence of a continuous bone marrow source. They suggested that a necessary condition is that each idiotype should recognize itself, i.e. should have a low affinity for binding itself. Although such antibodies have been described (Kang and Köhler, 1986), it seems much simpler to change to the parameter regime with higher rates of complex removal. This has the second advantage of the close correspondence between the model behavior (see Fig. 12) and the experimental data (Lundkvist et al., 1989; Varela et al., 1991).

This work was performed under the auspices of the U.S. Department of Energy. It was supported in part by NIH Grants AI28433 and RR06555 (A.P.), by NSF Grant CTS89-57213 (I.K.), and a Packard Foundation Fellowship (I.K.). It was also supported by the Santa Fe Institute through their Theoretical Immunology Program and by the Los Alamos National Laboratory Center for Nonlinear Studies.

\section{APPENDIX. NUMERICAL METHODS}

Bifurcation diagrams were computed using AUTO (Doedel. 1981). which is a special purpose software package for numerical bifurcation analysis. Nullclines, invariant manifolds and trajectories were computed by GRIND (De Boer. 1983). Limit cycles were continued using AUTO and by a shooting method, with the variational equations integrated using ODESSA (Leis and Kramer. 1988). Equilibrium states in the graphs were obtained by Newton-Raphson iteration implemented in GRIND. The accurate Poincare section of the intermittency was obtained with SCIGMA (Taylor et al.. 1990). The rough Poincare sections shown in bifurcation diagrams were obtained by checking the Poincare variable at each small time step of the integrator. Once a crossing of the section was detected the values of the variables at the section were estimated by a first-order linearization. This turned out to be sufficiently accurate for our graphical representations. Our procedure for generating multiple Poincare sections consists of the following steps. (1) Start close to an orbit and integrate the system for a long time (i.e. typically 5000 time steps) in order to attain an orbit. (2) Restart at the orbit attained and record 10 crossings of the Poincare section. (3) Restart at the last point, but interchange the variables with subscript 1 and 2 . in order to test whether another asymmetric attractor exists. Record another 10 crossings of the Poincare section. (4) Make a small change in the bifurcation parameter (i.e. typically $1 \%$ ). (5) Go back to step (1). We realize that this procedure may give an incomplete sample of the model's behavior. because not all forms of behavior need to be attained. 


\section{LITERATURE}

Andersson, J., A. Coutinho, W. Lernhardt and F. Melchers. 1977. Clonal growth and maturation to immunoglobulin secretion in vitro of every growth inducible B lymphocyte. Cell 10, 27-34.

Aronson, D. G., M. Golubitsky and J. Mallet-Paret. 1991. Ponies on a merry-go-round in large arrays of Josephson junctions. Nonlinearity 4. 903-910.

Berek, C. and C. Milstein. 1988. The dynamic nature of the antibody repertoire. Immunol. Rer. $105,5-26$.

Coutinho, A., A. Bandeira, P. Pereira, D. Portnoï. D. Holmberg. C. Martinez-A and A. Freitas. 1990. Selection of lymphocyte repertoires: The limits of clonal versus network organization. Symp. Quant. Biol., Cold Spring Harbor Lab. NY 54, 159-170.

Darnell. J. E.. H. Lodish and D. Baltimore. 1986. Molecular Cell Biology. pp. 1108-1109. New York: Scientific American Books.

De Boer. R. J. 1983. GRIND: Great Integrator Differential Equations. University of Utrecht, The Netherlands: Bioinformatics Group.

De Boer. R. J. 1988. Symmetric idiotypic networks: connectance and switching, stability, and suppression. In Theoretical Immunology-Part Two. A. S. Perelson (Ed.). pp. 265-289: SFI Studies in the Science of Complexity: Vol. III. Redwood City. CA: Addison-Wesley.

De Boer. R. J. and P. Hogeweg. 1989a. Stability of symmetric idiotypic networks - a critique of Hoffman's analysis. Bull. math. Biol. 51, 217-222.

De Boer. R. J. and P. Hogeweg. 1989b. Memory but no suppression in low-dimensional symmetric idiotypic networks. Bull. math. Biol. 51. 223-246.

De Boer. R. J. and P. Hogeweg. 1989c. Unreasonable implications of reasonable idiotypic network assumptions. Bull. math. Biol. 51, 381-408.

De Boer. R. J. and A. S. Perelson. (1991). Size and connectivity as emergent properties of a developing immune network. $J$. theor. biol. 149, 381-424.

De Boer. R. J.. I. G. Kevrekidis and A. S. Perelson. 1990. A simple idiotypic network model with complex dynamics. Chem. Engng Sci. 45. 2375-2382.

De Boer, R. J.. A. S. Perelson and I. G. Kevrekidis. 1993. Immune network behavior-I. From stationary states to limit cycle oscillations. Bull. math. Biol. 55. 745-780.

Doedel. E. J. 1981. AUTO: a program for the bifurcation analysis of autonomous systems. Cong. Num. 30, 265-285

Eisen, 1980. Immunolog1: 2nd edn. Hagerstown. MA: Harper and Row.

Gardner, M. R. and W. R. Ashby, 1970. Connectance of large dynamic (cybernetic) systems: critical values for stability. Nature 228. 784

Jerne. N. K. 1974. Towards a network theory of the immune system. Ann. Immunol. (Inst. Pasteur) 125 C. 373-389.

Kang. C.-Y. and H. Köhler. 1986. Immunoglobulin with complementary paratope and idiotope J. exp. Med. 163, 787-796.

Leis, J. R. and M. A. Kramer. 1988. ODESSA. an ordinary differential equation solver with explicit simultaneous sensitivity analysis. AC.M Trans. Math. Software 14, 61-67.

Lorenz, E. N. 1963. Deterministic nonperiodic flow. J. atmos. Sci. 20. 130-141.

Lundkvist. I. A. Coutinho. F. Varela and D. Holmberg. 1989. Evidence for a functional idiotypic network amongst natural antibodies in normal mice. Proc. natn. Acad. Sci. LSA 86. $5074-5078$.

May, R. M. 1972. Will a large complex system be stable? Nature 238, 413-414.

Neumann. A. U. and G. Weisbuch. 1991. Window automata analysis of population dynamics in the immune system. Bull. math. Biol. 54, 21-44.

Perelson, A. S. 1981. Receptor clustering on a cell surface. III. Theory of receptor crosslinking by multivalent ligands: Description by ligand states. Math. Biosci. 53, 1-39.

Perelson, A. S. and C. De Lisi. 1980. Receptor clustering on a cell surface. I. Theory of receptor cross-linking by ligands bearing two chemically identical functional groups. Math. Biosci. 48 , 71-110. 
Perelson, A. S and G. Weisbuch. 1992. Modeling immune reactivity in secondary lymphoid organs. Bull. math. Biol. 54. 649-672.

Pomeau. Y, and P. Manneville. 1980. Intermittent transition to turbulence in dissipative dynamical systems. Comm. math. Phys. 74, 189-197.

Segel, L. A. and A. S. Perelson. 1989. Shape space analysis of immune networks. In Cell to Cell Signalling: From Experiments to Theoretical Models. A. Goldbeter (Ed.), pp. 273-282. New York: Academic Press.

Sparrow, C. 1982. The Lorenz equations: bifurcations chaos and strange attractors. Applied Mathematical Sciences. Vol. 41. New York: Springer Verlag.

Sprent, J. 1989. T lymphocytes and the thymus. In Fundamental Immunology: W. E. Paul (Ed.). pp. 69-93. New York: Raven Press.

Stewart. J. and F. J. Varela. 1989. Exploring the meaning of connectivity in the immune network. Imminol. Rev. 110, 37-61.

Stewart, J. and F. J. Varela. 1990. Dynamics of a class of immune networks. II. Oscillatory activity of cellular and humoral components. J. theor. Biol. 144. 103-115.

Swift, J. W. and K. Wiesenfeld. 1984. Suppression of period doubling in symmetric systems Phis. Rev. Lett. 52, 705-708.

Taylor, M. A., M. S. Jolly and I. G. Kevrekidis. 1990. SCIG.MA. Technical Report. Department of Chemical Engineering. Princeton University.

Varela. F. J.. A. Anderson, G. Dietrich. A. Sundblad. D. Holmberg. M. Kazatchkine and A. Coutinho. 1991. The population dynamics of natural antibodies in normal and autoimmune individuals. Proc. natn. Acad. Sci. USA 88. 5917-5921.

Weisbuch. G.. R. J. De Boer and A. S. Perelson. 1990. Localized memories in idioty pic networks. J. theor. Biol. 146, 483-499.

Received 4 May 1992

Revised 20 August 1992 